\title{
Suppression of Basil Downy Mildew Caused by Peronospora belbahrii using Resistance Inducers, Mineral Salts and Anti-transpirants Combined with Different Rates of Nitrogen Fertilizer under Field Conditions Eman W.R. Ghebrial and M.G.A. Nada \\ Plant Pathol. Res. Inst., A. R. C., Giza, Egypt.
}

$\mathbf{F}$ ield experiments were carried out under natural infection of basil downy mildew in the Experimental Farm of Sids Agricultural Research Station, Agric. Res. Center, Beni-Sweif governorate during 2015 and 2016 growing seasons in order to evaluate the efficacy of different compounds of resistance inducers, mineral salts and antitranspirants applied on sweet basil plants (Balady variety) as foliar spray combined with two rates of nitrogen fertilizer for controlling basil downy mildew caused by Peronospora belbahrii. In general, all treatments had a positive effect on the reduction of disease severity with significant increase in fresh and dry weights of herb and essential oil yield at the end of experiment. The high efficacies of the tested compounds were observed when nitrogen was applied at the recommended rate. Chitosan $(0.05 \%)$, di-potassium phosphate (20 $\mathrm{mM})$ and potassium silicate $(20 \mathrm{mM})$ in addition to the fungicide Stone $50 \%$ were the most effective in this concern. Furthermore, plants sprayed with each of chitosan, di-potassium phosphate and potassium silicate showed significant increases in the defence-related enzymes, peroxidase (POD) and phenylalanine ammonia-lyase (PAL) as compared with the untreated basil plants.

Keywords: Anti-transpirants, chitosan, defence-related enzymes, dipotassium phosphate, downy mildew, mineral salts, nitrogen fertilizer, potassium silicate, resistance inducers and sweet basil.

Sweet basil (Ocimum basilicum L., Fam. Lamiaceae) is the most commercially important annual culinary herb crop used for both fresh and dry consumption and as a source of essential oils and oleoresin for manufacturing perfumes, food flavours and aromatherapy products (Simon et al., 1990). Basil is an important source of antioxidants (Koroch et al., 2010) and antimicrobial agents (Hussain et al., 2008) with a potential use in food preservation (Suppakul et al., 2003). Recently, a new disease of basil, downy mildew, incited by P. belbahrii Thines (Garibaldi et al., 2004; Belbahri et al., 2005 and Thines et al., 2009) was observed for the first time in Egypt especially in Beni-Sueif governorate in 2013 (Hilal and Ghebrial, 2014). Since then the disease has been quickly spread to all the Egyptian basil-growing areas.

The epidemiology of the pathogen is still scanty. However, it is believed that the pathogen has spread globally in recent years via the shipment of infested seed as well as through wind currents (Garibaldi et al., 2004 and McGrath, 2009). Under 
favorable conditions, the disease rate could reach $100 \%$ within three to five days after the attack, making prevention and control extremely difficult. Chemical control is considered one of the most effective ways to protect many crops from downy mildew infection (Gullino et al., 2009) but this is complicated by the very limited availability of chemicals for basil crop as medicinal, due to the risk of the presence of residues at harvest as well as the difficulty to obtain fungicide registration for minor crops such as basil (Leadbeater and Gisi, 2010). Protectant fungicides may not provide control once the pathogen has infected the plants and begins sporulating on the abaxial surface of infected leaves (Gregory, 2009). However, there are reports on the appearance of resistant isolates to the fungicide mefenoxamand some isolates showed reduced sensitivity to dimethomorph and mandipropamid (Cohen et al., 2013 and Ben-Naim et al., 2015). In Egypt, the unavailability of commercially resistant basil varieties intensifies the need for alternative methods for disease control. One of the potential methods of reducing the severity of the disease in an environmentally safe manner is the induction of plant resistance. Certain substances, such as salicylic acid, bion, silicon, potassium salts were reported to induce resistance in basil plants against downy mildew (Mersha et al., 2012; Gilardi et al., 2013, 2015; Homa et al., 2014 and Patel et al., 2014).

Mono- and di-potassium salts of phosphorous acid provided the best control of basil downy mildew, whereas moderate disease suppression was provided by mandipropamid, cyazofamid and fiuopicolide (Homa et al., 2014). It was speculated that basic phosphates applied to plants could sequester apoplastic calcium, altering membrane integrity and influencing the activity of apoplastic enzymes like polygalacturonases, thereby releasing elicitor-active oligogalacturonides from plant cell walls (Walters and Murray, 1992). Phosphate-mediated induction of resistance was associated with increased activities of phenylalanine ammonia-lyase (PAL), peroxidase (POD) and lipoxygenase (Mitchell and Walters, 2004).

Acibenzolar-S-methyl (ASM) has been developed as a potent systemic acquired resistance (SAR) activator which does not has antimicrobial properties, but instead increases crop resistance to diseases by activation the SAR signal transduction pathway in several plant species (Lopez and Lucas, 2002 and Baysal et al., 2003). The area under the disease progress curve (AUDPC) of basil downy mildew disease severity was significantly reduced compared to the non-treated control when ASM was sprayed or drenched pre-, or pre- + post-inoculation at rates of 25-400 mg/l. Basil plants treated with ASM and challenged with the pathogen showed significantly higher peroxidase activity than the non-treated control at 8 days after inoculation (Mersha et al., 2013).

Although silicon ( $\mathrm{Si}$ ) is not listed among the essential elements, its role in conferring plant resistance to both abiotic and biotic stresses has received increasing attention (Liang et al., 2003 and Epstein, 2009). There is a cumulative body of evidence linking the presence of $\mathrm{Si}$ with resistance of plants against fungal pathogens (Kanto et al., 2006 and Dallagnol et al., 2012). However, the mechanisms by which $\mathrm{Si}$ provides protection against fungal plant pathogens are still unclear. Some authors believe that $\mathrm{Si}$ acts as a physical barrier in cell walls, preventing the penetration of fungal hyphae into host tissues (Samuels et al., 1991 and Bowen et al., 
1992), while others believe that $\mathrm{Si}$ is related to specific plant defence reactions (Fawe et al., 2001 and Rodrigues et al., 2004).

Among the most promising bioactive oligosaccharides is chitosan which has attracted attention because of its unique biological properties, including its inhibitory effect on the growth of various pathogenic fungi, its ability to be a potent elicitor of plant defense reactions and as an agent for plant growth (Prapagdee et al., 2007). Chitosan has been reported to protect different plant species against downy mildew (Manjunatha et al., 2008 and El-Mougy et al., 2014). Epidermis-coating polymers, such as film forming anti-transpirants, have been reported to provide protection against several foliar plant diseases (Nasraoui et al., 1996 and Haggag, 2002). Although the effect of anti-transpirants on disease control appears similar to those of the natural cuticle layer in defending plant pathogens (Hsieh and Huang, 1999), physical effects should be also considered (Nasraoui, 1993). Nitrogen (N) is essential for plant growth and development, and crop plants will obtain it from the soil as nitrate or ammonium (Lea and Azevedo, 2006 and Lea et al., 2007). Application of $\mathrm{N}$ fertilizer above recommended rates can lead to significantly higher downy mildew disease severity (Zarafi et al., 2005 and dos Santos et al., 2009).

The objective of this study was to evaluate the efficacy of different plant resistance inducers, mineral salts and film forming anti-transpirants applied on basil as foliar spray combined with two rates of nitrogen fertilizer for controlling basil downy mildew under field condition.

\section{Materials and Methods}

A field experiment was carried out under natural infection of basil downy mildew in the Experimental Farm of Sids Agricultural Research Station, Agric. Res. Center, Beni-Sweif governorate during the two growing seasons of 2015 and 2016. The soil texture is clay ( $16.5 \%$ sand, $30.1 \%$ silt, $53.4 \%$ clay, $\mathrm{pH}$ of 8.1 , EC 1.2 $\mathrm{dSm}^{-1} ; 1.3 \%$ organic matter and 26.2, 10.1 and $176 \mathrm{ppm}$ available $\mathrm{N}, \mathrm{P}$ and $\mathrm{K}$, respectively). During both trial years, the following treatments were applied as foliar spray:

- Chitosan at a concentration of $0.05 \%$ dissolved in $0.01 \mathrm{M}$ acetic acid and adjusted $\mathrm{pH}$ to 5.6 using $1 \mathrm{~N} \mathrm{NaOH}$.

- Salicylic acid at the rate of $25 \mathrm{mML}^{-1}$, added with $0.01 \%$ tween 80 .

- Bion (Acibenzolar-S-methyl) at the rate of $50 \mathrm{mg} \mathrm{L}^{-1}$.

- Mono-potassium phosphate $\mathrm{K}_{2} \mathrm{HPO}_{4}$ at the rate of $20 \mathrm{mML}^{-1}$.

- Di- potassium phosphate $\mathrm{KH}_{2} \mathrm{PO}_{4}$ at the rate of $20 \mathrm{mML}^{-1}$.

- Potassium carbonate $\mathrm{K}_{2} \mathrm{CO}_{3}$ at the rate of $20 \mathrm{mML}^{-1}$.

- Potassium silicate $\mathrm{K}_{2} \mathrm{O}_{3} \mathrm{Si}$ at the rate of $20 \mathrm{mML}^{-1}$.

- Silicon dioxide $\mathrm{SiO}_{2}$ at the rate of $10 \mathrm{mML}^{-1}$.

- Kaolin (Aluminum silicate, $\mathrm{Al}_{2} \mathrm{SiO}_{5}$ ) at the rate of $1 \%$.

- The commercial antitranspirant product Green miracle at the rate of $3 \mathrm{mlL}^{-1}$ water.

- The fungicide Stone 50\% (Dimethomorph $50 \%$ ) at the rate of $50 \mathrm{gm} / 100 \mathrm{~L}$ water.

- Control (untreated). 
In each season, the soil was mechanically ploughed and planked twice. During the preparation for cultivation, calcium super-phosphate $\left(15.5 \% \mathrm{P}_{2} \mathrm{O}_{5}\right)$ was added at the rate of $200 \mathrm{~kg} / \mathrm{fed}$. The uniform healthy basil seedlings (Balady variety), 10-15 $\mathrm{cm}$ length were transplanted on rows at $15 \mathrm{~cm}$ spacing between plants. Weeds were removed by manual operations as needed and plants were irrigated regularly as necessary, throughout the growing season in order to maintain constant growth.

Nitrogen was applied in the form of ammonium sulphate $(20.6 \% \mathrm{~N})$, at the rate of 400 (recommended rate), $500 \mathrm{~kg} / \mathrm{fed}$. (High rate) as follow: the first one was added after 21 days from transplanting and the second after 30 days from the first application. The remainder amounts were added after each cut. Potassium sulphate $\left(48 \% \mathrm{~K}_{2} \mathrm{O}\right)$ was added at the rate of $75 \mathrm{~kg} / \mathrm{fed}$. The plants were sprayed weekly, always performed early in the morning, with the tested compounds after 21 days from transplanting (before the appearance of first symptoms) until run off.

The experiment was arranged in a split-split plot design, with three replications. Monitoring and scouting the plants weekly for downy mildew and disease incidence and severity were estimated as follows:

Disease incidence:

Percentage of disease incidence was recorded as the number of diseased plants relative to the number of growing plants for each treatment, and then the average of disease incidence was calculated. Protection was calculated using the formula:

Reduction of downy mildew disease incidence $(\%)=[\mathrm{C}-\mathrm{T}] / \mathrm{C} \times 100$

Whereas: $\mathrm{C}$ and $\mathrm{T}$ are percentage of downy mildew incidence in control and treated plants, respectively.

\section{Disease severity:}

Disease severity was measured according to (Abd-Alla, 2004). Percentage of disease severity was recorded according to the following equation:

Disease severity $\%=\left[\sum(\mathrm{n} \times \mathrm{c})\right] /(\mathrm{N} \times \mathrm{C}) \times 100$

Whereas: $\mathrm{n}=$ Number of infected leaves, $\mathrm{c}=$ Category number, $\mathrm{N}=$ Total number of examined leaves and $\mathrm{C}=$ The highest category number of infection.

The plants were harvested three times (first, second and third cuttings) when the basil plants had full flowers by cutting plants $10 \mathrm{~cm}$ above the soil surface with three replications in each cut. Fresh and dry weights of herb yield (ton/fed.) were determined.

To determine the yield of essential oil, the fresh plants (leaves and flowers) were collected from chitosan, di-potassium phosphate, potassium silicate and fungicide treatments during the three cuts and weighted (100g/treatment) representing each replicate then subjected to steam distillation and determined according to Guenther (1961).

Peroxidase activity was determined using the method described in the Worthington enzyme manual (Worthington, 1971). Phenylalanine ammonia-lyase enzyme was determined according to Zucker (1965). 
Data were statistically analyzed for computing L.S.D. test at $5 \%$ probability according to the procedure outlined by Snedecor and Cochran (1989).

\section{R e s u l t s}

According to the results obtained of the two growing seasons it was found that the three tested elicitors (bion, chitosan and salicylic acid) in addition to the fungicide Stone $50 \%$ significantly provided protection against downy mildew infection and affected disease severity at an important level at the two nitrogen rates compared to the untreated control for the three cuts of plants (Tables, 1 and 2). In general, the high efficacy values of the tested elicitors were observed when the nitrogen was applied at the recommended rate $(400 \mathrm{~kg} / \mathrm{fed}$.). The highest reduction in disease incidence was resulted from using any of the fungicide Stone $50 \%$ or chitosan treatments with significant differences between them at the two nitrogen rates, followed by salicylic acid treatment. The reduction in disease incidence and disease severity percentages obtained with the fungicide Stone $50 \%$ in the first season (2015) were $97.8 \& 1.2 \%$ for the first cut, $89.2 \& 8.1 \%$ for the second cut and $91.3 \& 2.1 \%$ for the third cut, respectively at the recommended rate of nitrogen (400 kg/fed.). Meanwhile application with fungicide in replicates gave high nitrogen fertilizer rate (500 kg/fed.) resulted in $95.0 \& 2.5 \%, 85.5 \& 14.0 \%$ and $91.0 \& 3.9 \%$, respectively in percentage of reduction in disease incidence and disease severity. While, they recorded $(100.0 \& 0.0 \%),(92.5 \& 7.0 \%)$ and $(96.03 \& 1.57 \%)$, respectively at the recommended rate of nitrogen and $(98.7 \& 1.2 \%),(87.3 \& 10.7 \%)$ and $(93.80 \& 2.00 \%)$, respectively at the high rate of nitrogen fertilizer during the second growing season (2016). On the other hand, reduction in percentages of disease incidence and value of disease severity were (95.8 \& 3.1\%), (85.0 \& 12.6\%), $(89.3 \& 5.9 \%)$ and $(93.70 \& 4.0 \%),(83.5 \& 14.8 \%),(89.0 \& 7.2 \%)$ in the first growing season and $(99.03 \& 2.5 \%),(89.9 \& 9.4 \%),(95.0 \& 3.1 \%)$ and $(96.2 \&$ $2.9 \%),(85.7 \& 11.5 \%),(92.4 \& 5.9 \%)$ in the second growing season due to chitosan treatment for the three plant cuts, respectively at the two nitrogen levels. Bion showed relatively less effect in this concern.

Results presented in Tables 3 and 4 exhibit that the three elicitors tested improved plant growth as shown by the significant increments in fresh and dry weights of herb (ton/fed.) in the two experimental seasons compared to the untreated control. Significant differences among treatments were found for plant weights. In general, the plant weights were significantly affected by the rate of nitrogen fertilizer added. Application of high amount of nitrogen fertilizer significantly increased fresh weight of herb but decreased dry weight of herb. 
Table 1. Reduction in percentage of disease incidence due to different elicitors applied as foliar spray at two nitrogen rates against downy mildew of basil for three plant cuts during the two growing seasons 2015 and 2016 under field conditions

\begin{tabular}{|c|c|c|c|c|c|c|c|c|c|c|c|}
\hline \multirow[b]{2}{*}{ Treatment } & \multirow[b]{2}{*}{$\begin{array}{l}\text { N. rates } \\
\text { (kg/fed.) }\end{array}$} & \multicolumn{5}{|c|}{ Season 2015} & \multicolumn{5}{|c|}{ Season 2016} \\
\hline & & $\begin{array}{l}1^{\mathrm{st}} \\
\mathrm{cut}\end{array}$ & $\begin{array}{l}2^{\text {nd }} \\
\text { cut }\end{array}$ & $\begin{array}{l}3^{\text {rd }} \\
\text { cut }\end{array}$ & Mean & $\begin{array}{c}\text { Overall } \\
\text { mean }\end{array}$ & $\begin{array}{l}1^{\text {st }} \\
\text { cut }\end{array}$ & $\begin{array}{l}2^{\text {nd }} \\
\text { cut }\end{array}$ & $\begin{array}{l}3^{\text {rd }} \\
\text { cut }\end{array}$ & Mean & $\begin{array}{c}\text { Overall } \\
\text { mean }\end{array}$ \\
\hline \multirow{2}{*}{ Bion } & 400 & 60.2 & 46.5 & 52.5 & 53.1 & \multirow{3}{*}{52.5} & 64.5 & 48.8 & 54.7 & 56.0 & \multirow{3}{*}{54.8} \\
\hline & 500 & 59.8 & 44.3 & 51.9 & 52.0 & & 62.1 & 46.7 & 52.0 & 53.6 & \\
\hline \multicolumn{2}{|c|}{ Mean } & 60.0 & 45.4 & 52.2 & --- & & 63.3 & 47.7 & 53.3 & --- & \\
\hline \multirow{2}{*}{ Chitosan } & 400 & 95.8 & 85.0 & 89.3 & 90.0 & \multirow{3}{*}{89.4} & 99.1 & 89.9 & 95.0 & 94.6 & \multirow{3}{*}{93.0} \\
\hline & 500 & 93.7 & 83.5 & 89.0 & 88.7 & & 96.2 & 85.7 & 92.4 & 91.4 & \\
\hline \multicolumn{2}{|c|}{ Mean } & 94.7 & 84.3 & 89.1 & --- & & 97.6 & 87.8 & 93.7 & --- & \\
\hline \multirow{2}{*}{$\begin{array}{l}\text { Salicylic } \\
\text { acid }\end{array}$} & 400 & 87.2 & 78.6 & 83.0 & 82.9 & \multirow{3}{*}{80.5} & 91.4 & 82.6 & 86.5 & 86.8 & \multirow{3}{*}{83.6} \\
\hline & 500 & 85.1 & 72.7 & 76.5 & 78.1 & & 87.0 & 75.9 & 78.2 & 80.3 & \\
\hline \multicolumn{2}{|c|}{ Mean } & 86.1 & 75.7 & 79.7 & --- & & 89.2 & 79.2 & 82.3 & --- & \\
\hline \multirow{2}{*}{ Stone $50 \%$} & 400 & 97.8 & 89.3 & 91.3 & 92.8 & \multirow{3}{*}{91.7} & 100.0 & 92.5 & 96.0 & 96.2 & \multirow{3}{*}{94.7} \\
\hline & 500 & 95.1 & 85.5 & 91.0 & 90.5 & & 98.7 & 87.3 & 93.8 & 93.3 & \\
\hline \multicolumn{2}{|c|}{ Mean } & 96.4 & 87.4 & 91.2 & --- & & 99.3 & 89.9 & 94.9 & --- & \\
\hline \multirow{2}{*}{ Control } & 400 & 0.0 & 0.0 & 0.0 & 0.0 & \multirow{3}{*}{0.0} & 0.0 & 0.0 & 0.0 & 0.0 & \multirow{3}{*}{0.00} \\
\hline & 500 & 0.0 & 0.0 & 0.0 & 0.0 & & 0.0 & 0.0 & 0.0 & 0.0 & \\
\hline \multicolumn{2}{|c|}{ Mean } & 0.0 & 0.0 & 0.0 & --- & & 0.0 & 0.0 & 0.0 & --- & \\
\hline \multirow{2}{*}{$\begin{array}{l}\text { Mean of } \\
\text { nitrogen } \\
\text { rates }\end{array}$} & 400 & 68.2 & 59.9 & 63.2 & 63.8 & \multirow{3}{*}{--- } & 71.0 & 62.8 & 66.4 & 66.7 & \multirow{3}{*}{---} \\
\hline & 500 & 66.7 & 57.2 & 61.7 & 61.9 & & 68.8 & 59.1 & 63.3 & 63.7 & \\
\hline \multicolumn{2}{|c|}{ Overall mean } & 67.5 & 58.5 & 62.4 & --- & & 69.9 & 60.9 & 64.9 & --- & \\
\hline \multicolumn{3}{|c|}{$\begin{array}{l}\text { L.S.D. at } 5 \% \text { for: } \\
\text { Treatments }(\mathrm{T})=0.55 \\
\text { Nitrogen rates }(\mathrm{N})=0.15 \\
\text { Cuttings }(\mathrm{C})=0.18\end{array}$} & \multicolumn{3}{|c|}{$\begin{array}{ll}\mathrm{T} \times \mathrm{N} & =0.33 \\
\mathrm{~T} \times \mathrm{C} & =0.41 \\
\mathrm{~N} \times \mathrm{C} & =0.26 \\
\mathrm{~T} \times \mathrm{N} \times \mathrm{C} & =0.57\end{array}$} & & \multicolumn{2}{|c|}{$\begin{array}{l}\mathrm{T}=0.56 \\
\mathrm{~N}=0.14 \\
\mathrm{C}=0.17\end{array}$} & \multicolumn{2}{|c|}{$\begin{array}{ll}\mathrm{T} \times \mathrm{N} & =0.31 \\
\mathrm{~T} \times \mathrm{C} & =0.38 \\
\mathrm{~N} \times \mathrm{C} & =0.24 \\
\mathrm{~T} \times \mathrm{N} \times \mathrm{C} & =0.54\end{array}$} & \\
\hline
\end{tabular}

Among elicitors tested with recommended rate of nitrogen fertilizer (400 kg/fed.), chitosan was the most effective treatment in this respect. It yielded the highest mean of fresh and dry weight of herb through the three cuts during the season 2015, being 9.78and 2.12 tons/fed., respectively. Meanwhile, the same trend was also noticed through the three cuts determined in the growing season 2016, being 10.84 and 2.35 tons/fed. on the average, respectively. Increasing rate of nitrogen fertilizer to 500 $\mathrm{kg} / \mathrm{fed}$. caused significant increases in herb fresh weight with all elicitors tested. The highest means of herb fresh weight were recorded from chitosan treatment. The corresponding means were 10.31 and 11.08 tons/fed. during the two successive seasons (2015 and 2016), respectively. On the other hand, salicylic acid treatment showed moderate effect whereas; bion treatment gave the lowest increase in herb fresh and dry weights during the two growing seasons. 
Table 2. Evaluation of different elicitors applied as foliar spray at two nitrogen rates on disease severity of basil downy mildew for three plant cuts during the two growing seasons 2015 and 2016 under field conditions

\begin{tabular}{|c|c|c|c|c|c|c|c|c|c|c|c|}
\hline \multirow{3}{*}{ Treatment } & \multirow{3}{*}{$\begin{array}{c}\text { Nitrogen } \\
\text { rates } \\
(\mathrm{kg} / \mathrm{fed} .)\end{array}$} & \multicolumn{10}{|c|}{ Disease severity \% } \\
\hline & & \multicolumn{5}{|c|}{ Season 2015} & \multicolumn{5}{|c|}{ Season 2016} \\
\hline & & $\begin{array}{l}1^{\text {st }} \\
\text { cut }\end{array}$ & $\begin{array}{l}2^{\text {nd }} \\
\text { cut }\end{array}$ & $\begin{array}{l}3^{\text {rd }} \\
\text { cut }\end{array}$ & Mean & $\begin{array}{l}\text { Overall } \\
\text { mean }\end{array}$ & $\begin{array}{l}1^{\mathrm{st}} \\
\mathrm{cut}\end{array}$ & $\begin{array}{l}2^{\text {nd }} \\
\text { cut }\end{array}$ & $\begin{array}{l}3^{\text {rd }} \\
\text { cut }\end{array}$ & Mean & $\begin{array}{c}\text { Overall } \\
\text { mean }\end{array}$ \\
\hline \multirow{2}{*}{ Bion } & 400 & 19.3 & 36.9 & 22.7 & 26.3 & \multirow{3}{*}{27.3} & 16.1 & 35.8 & 20.3 & 24.1 & \multirow{3}{*}{25.2} \\
\hline & 500 & 20.6 & 37.9 & 26.4 & 28.3 & & 18.0 & 36.2 & 24.8 & 26.3 & \\
\hline \multicolumn{2}{|c|}{ Mean } & 19.9 & 37.4 & 24.5 & --- & & 17.0 & 36.0 & 22.5 & --- & \\
\hline \multirow{2}{*}{ Chitosan } & 400 & 3.1 & 12.6 & 5.9 & 7.2 & \multirow{3}{*}{7.9} & 2.5 & 9.4 & 3.1 & 5.0 & \multirow{3}{*}{5.9} \\
\hline & 500 & 4.0 & 14.8 & 7.2 & 8.7 & & 2.9 & 11.5 & 5.9 & 6.8 & \\
\hline \multicolumn{2}{|c|}{ Mean } & 3.6 & 13.7 & 6.6 & --- & & 2.7 & 10.5 & 4.5 & --- & \\
\hline \multirow{2}{*}{$\begin{array}{c}\text { Salicylic } \\
\text { acid }\end{array}$} & 400 & 4.3 & 17.9 & 10.1 & 10.8 & \multirow{3}{*}{11.5} & 3.0 & 15.2 & 7.8 & 8.7 & \multirow{3}{*}{9.6} \\
\hline & 500 & 5.3 & 20.0 & 11.1 & 12.1 & & 3.9 & 18.0 & 9.8 & 10.6 & \\
\hline \multicolumn{2}{|c|}{ Mean } & 4.8 & 18.9 & 10.6 & --- & & 3.5 & 16.6 & 8.8 & --- & \\
\hline Stone & 400 & 1.2 & 8.1 & 2.2 & 3.8 & \multirow{3}{*}{5.3} & 0.0 & 7.0 & 1.6 & 2.9 & \multirow{3}{*}{3.8} \\
\hline $50 \%$ & 500 & 2.5 & 14.0 & 3.9 & 6.8 & & 1.2 & 10.7 & 2.0 & 4.6 & \\
\hline \multicolumn{2}{|c|}{ Mean } & 1.9 & 11.0 & 3.0 & --- & & 0.6 & 8.9 & 1.8 & --- & \\
\hline \multirow{2}{*}{ Control } & 400 & 48.6 & 63.1 & 54.3 & 55.3 & \multirow{3}{*}{57.5} & 50.9 & 66.5 & 55.2 & 57.5 & \multirow{3}{*}{60.3} \\
\hline & 500 & 51.7 & 70.8 & 56.5 & 59.7 & & 55.2 & 74.3 & 59.7 & 63.1 & \\
\hline \multicolumn{2}{|c|}{ Mean } & 50.2 & 66.9 & 55.4 & --- & & 53.1 & 70.4 & 57.4 & --- & \\
\hline \multirow{2}{*}{$\begin{array}{l}\text { Mean of } \\
\text { nitrogen }\end{array}$} & 400 & 15.3 & 27.7 & 19.0 & 20.7 & \multirow{3}{*}{--- } & 14.5 & 26.8 & 17.6 & 19.6 & \\
\hline & 500 & 16.8 & 31.5 & 21.0 & 23.1 & & 16.2 & 30.2 & 20.4 & 22.3 & --- \\
\hline \multicolumn{2}{|c|}{ Overall mean } & 16.1 & 29.6 & 20.0 & --- & & 15.4 & 28.5 & 19.02 & --- & \\
\hline \multicolumn{2}{|c|}{ L.S.D. at $5 \%$ for: } & & $\mathrm{Tx}$ & - & & & \multicolumn{4}{|c|}{$\mathrm{T} \times \mathrm{N}=0.29$} & \\
\hline Treatmen & ts $(\mathrm{T})$ & $=0.28$ & $\mathrm{Tx}$ & - & .36 & & $\mathrm{~T}=0$ & 1 & & $=0.35$ & \\
\hline Nitrogen & $\operatorname{rates}(\mathrm{N})=$ & $=0.13$ & $\mathrm{Nx}$ & - & .23 & & $\mathrm{~N}=0$ & 3 & $\mathrm{C}$ & 0.22 & \\
\hline Cuttings & ( C ) & $=0.16$ & $\mathrm{Tx}$ & $\times C=$ & .51 & & $\mathrm{C}=0$. & $\mathrm{T}$ & $\mathrm{N} \times \mathrm{C}$ & $=0.49$ & \\
\hline
\end{tabular}


Table 3. Effect of three elicitors applied as foliar spray at two nitrogen rates on fresh weight of herb (ton/fed.) for three cuts of basil plants naturally infected by downy mildew during two growing seasons 2015 and 2016 under field conditions

\begin{tabular}{|c|c|c|c|c|c|c|c|c|c|c|c|}
\hline \multirow{3}{*}{ Treatment } & \multirow{3}{*}{$\begin{array}{l}\text { N. rates } \\
(\mathrm{kg} / \mathrm{fed} .)\end{array}$} & \multicolumn{10}{|c|}{ Fresh weight of herb (ton/fed.) } \\
\hline & & \multicolumn{5}{|c|}{ Season 2015} & \multicolumn{5}{|c|}{ Season 2016} \\
\hline & & $\begin{array}{l}1^{\mathrm{st}} \\
\text { cut }\end{array}$ & $\begin{array}{l}2^{\text {nd }} \\
\text { cut }\end{array}$ & $\begin{array}{l}3^{\text {rd }} \\
\text { cut }\end{array}$ & Mean & $\begin{array}{c}\text { Overall } \\
\text { mean }\end{array}$ & $\begin{array}{l}1^{\mathrm{st}} \\
\text { cut }\end{array}$ & $\begin{array}{l}2^{\text {nd }} \\
\text { cut }\end{array}$ & $\begin{array}{l}3^{\text {rd }} \\
\text { cut }\end{array}$ & Mean & $\begin{array}{c}\text { Overall } \\
\text { mean }\end{array}$ \\
\hline \multirow{2}{*}{ Bion } & 400 & 4.7 & 6.2 & 7.3 & 6.1 & \multirow{3}{*}{6.3} & 5.2 & 6.6 & 8.0 & 6.6 & \multirow{3}{*}{6.8} \\
\hline & 500 & 4.9 & 6.6 & 7.9 & 6.5 & & 5.5 & 7.1 & 8.4 & 7.0 & \\
\hline \multicolumn{2}{|c|}{ Mean } & 4.8 & 6.4 & 7.6 & --- & & 5.4 & 6.8 & 8.2 & --- & \\
\hline \multirow{2}{*}{ Chitosan } & 400 & 7.8 & 9.7 & 11.8 & 9.8 & \multirow{3}{*}{10.0} & 8.7 & 10.7 & 13.1 & 10.8 & \multirow{3}{*}{11.0} \\
\hline & 500 & 8.3 & 10.2 & 12.4 & 10.3 & & 9.0 & 11.0 & 13.3 & 11.1 & \\
\hline \multicolumn{2}{|c|}{ Mean } & 8.0 & 10.0 & 12.1 & --- & & 8.8 & 10.8 & 13.2 & --- & \\
\hline \multirow{2}{*}{ Salicylic acid } & 400 & 7.0 & 8.9 & 11.0 & 9.0 & \multirow{3}{*}{9.3} & 7.8 & 10.1 & 12.1 & 10.0 & \multirow{3}{*}{10.2} \\
\hline & 500 & 7.6 & 9.5 & 11.6 & 9.6 & & 8.2 & 10.6 & 12.5 & 10.4 & \\
\hline \multicolumn{2}{|c|}{ Mean } & 7.3 & 9.2 & 11.3 & --- & & 8.0 & 10.3 & 12.3 & --- & \\
\hline \multirow{2}{*}{ Stone $50 \%$} & 400 & 8.1 & 10.2 & 12.4 & 10.2 & \multirow{3}{*}{10.5} & 8.9 & 11.0 & 13.4 & 11.1 & \multirow{3}{*}{11.3} \\
\hline & 500 & 8.7 & 10.6 & 12.9 & 10.7 & & 9.5 & 11.6 & 13.6 & 11.6 & \\
\hline \multicolumn{2}{|c|}{ Mean } & 8.4 & 10.4 & 12.7 & --- & & 9.2 & 11.3 & 13.5 & --- & \\
\hline \multirow{2}{*}{ Control } & 400 & 3.2 & 3.7 & 4.4 & 3.8 & \multirow{3}{*}{3.9} & 2.9 & 3.4 & 4.1 & 3.5 & \multirow{3}{*}{3.7} \\
\hline & 500 & 3.4 & 3.9 & 4.7 & 4.0 & & 3.3 & 3.8 & 4.4 & 3.8 & \\
\hline \multicolumn{2}{|c|}{ Mean } & 3.3 & 3.8 & 4.5 & --- & & 3.1 & 3.6 & 4.3 & --- & \\
\hline Mean of & 400 & 6.1 & 7.7 & 9.4 & 7.7 & \multirow{3}{*}{--- } & 6.7 & 8.3 & 10.1 & 8.4 & \multirow{3}{*}{--- } \\
\hline nitrogen rates & 500 & 6.9 & 8.2 & 9.9 & 8.2 & & 7.1 & 8.8 & 10.4 & 8.8 & \\
\hline \multicolumn{2}{|c|}{ Overall mean } & 6.4 & 8.0 & 9.6 & --- & & 6.9 & 8.6 & 10.29 & --- & \\
\hline \multicolumn{2}{|c|}{ L.S.D. at $5 \%$ for: } & \multicolumn{2}{|c|}{$\mathrm{T} \times \mathrm{N}$} & \multicolumn{3}{|l|}{$=\mathrm{ns}$} & & & $\mathrm{x} \mathrm{N}$ & $=\mathrm{ns}$ & \\
\hline Treatments ( & $=0.17$ & & & $=0.39$ & & & $\mathrm{~T}=0$ & & $\mathrm{x} \mathrm{C}$ & $=0.37$ & \\
\hline Nitrogen rate & $(\mathrm{N})=0.14$ & & & $=\mathrm{ns}$ & & & $\mathrm{N}=\mathrm{C}$ & & $\mathrm{XC}$ & $=\mathrm{ns}$ & \\
\hline Cuttings ( C & $=0.18$ & & $\mathrm{~N} \times \mathrm{C}$ & $=\mathrm{ns}$ & & & $\mathrm{C}=\mathrm{C}$ & & $\mathrm{x} \mathrm{N} \times \mathrm{C}$ & $=\mathrm{ns}$ & \\
\hline
\end{tabular}


Table 4. Effect of three elicitors applied as foliar spray at two nitrogen rates on dry weight of herb (ton/fed.) for three cuts of basil plants naturally infected by downy mildew during two growing seasons 2015 and 2016 under field conditions

\begin{tabular}{|c|c|c|c|c|c|c|c|c|c|c|c|}
\hline \multirow{3}{*}{ Treatment } & \multirow{3}{*}{$\begin{array}{l}\text { N. rates } \\
(\mathrm{kg} / \text { fed. })\end{array}$} & \multicolumn{10}{|c|}{ Herb dry weight (ton/fed.) } \\
\hline & & \multicolumn{5}{|c|}{ Season 2015} & \multicolumn{5}{|c|}{ Season 2016} \\
\hline & & $\begin{array}{l}1^{\text {st }} \\
\text { cut }\end{array}$ & $\begin{array}{l}2^{\text {nd }} \\
\text { cut }\end{array}$ & $\begin{array}{l}3^{\text {rd }} \\
\text { cut }\end{array}$ & Mean & \begin{tabular}{|c|} 
Overall \\
mean
\end{tabular} & $\begin{array}{l}1^{\text {st }} \\
\text { cut }\end{array}$ & $\begin{array}{l}2^{\text {nd }} \\
\text { cut }\end{array}$ & $\begin{array}{l}3^{\text {rd }} \\
\text { cut }\end{array}$ & Mean & $\begin{array}{l}\text { Overall } \\
\text { mean }\end{array}$ \\
\hline \multirow{2}{*}{ Bion } & 400 & 1.1 & 1.3 & 1.5 & 1.3 & \multirow{3}{*}{1.25} & 1.2 & 1.5 & 1.7 & 1.5 & \multirow{3}{*}{1.4} \\
\hline & 500 & 1.0 & 1.2 & 1.3 & 1.2 & & 1.1 & 1.3 & 1.5 & 1.3 & \\
\hline \multicolumn{2}{|c|}{ Mean } & 1.1 & 1.2 & 1.4 & $\begin{array}{ll}-- \\
--\end{array}$ & & 1.2 & 1.4 & 1.6 & $\begin{array}{c}-- \\
--\end{array}$ & \\
\hline \multirow{2}{*}{ Chitosan } & 400 & 1.9 & 2.1 & 2.4 & 2.1 & \multirow{3}{*}{2.06} & 2.1 & 2.3 & 2.6 & 2.3 & \multirow{3}{*}{2.3} \\
\hline & 500 & 1.8 & 2.0 & 2.2 & 2.0 & & 2.0 & 2.1 & 2.3 & 2.2 & \\
\hline \multicolumn{2}{|c|}{ Mean } & 1.8 & 2.0 & 2.3 & --- & & 2.2 & 2.2 & 2.5 & -- & \\
\hline \multirow{2}{*}{$\begin{array}{c}\text { Salicylic } \\
\text { acid }\end{array}$} & 400 & 1.7 & 2.0 & 2.2 & 2.0 & \multirow{3}{*}{1.89} & 1.9 & 2.2 & 2.4 & 2.2 & \multirow{3}{*}{2.1} \\
\hline & 500 & 1.6 & 1.8 & 2.0 & 1.8 & & 1.7 & 2.0 & 2.2 & 2.0 & \\
\hline \multicolumn{2}{|c|}{ Mean } & 1.6 & 1.9 & 2.1 & --- & & 1.8 & 2.1 & 2.3 & --- & \\
\hline \multirow{2}{*}{ Stone $50 \%$} & 400 & 2.0 & 2.3 & 2.5 & 2.3 & \multirow{3}{*}{2.16} & 2.2 & 2.4 & 2.7 & 2.4 & \multirow{3}{*}{2.4} \\
\hline & 500 & 1.8 & 2.1 & 2.3 & 2.0 & & 2.0 & 2.3 & 2.5 & 2.3 & \\
\hline \multicolumn{2}{|c|}{ Mean } & 1.9 & 2.1 & 2.4 & --- & & 2.1 & 2.4 & 2.6 & --- & \\
\hline \multirow{2}{*}{ Control } & 400 & 0.8 & 1.0 & 1.1 & 1.0 & \multirow{3}{*}{0.86} & 0.8 & 0.9 & 1.1 & 0.9 & \multirow{3}{*}{0.8} \\
\hline & 500 & 0.7 & 0.8 & 0.8 & 0.7 & & 0.6 & 0.7 & 0.7 & 0.7 & \\
\hline \multicolumn{2}{|c|}{ Mean } & 0.7 & 0.9 & 0.9 & --- & & 0.7 & 0.8 & 0.9 & --- & \\
\hline \multirow{2}{*}{$\begin{array}{c}\text { Mean of } \\
\text { nitrogen } \\
\text { rates }\end{array}$} & 400 & 1.5 & 1.7 & 1.9 & 1.7 & \multirow{3}{*}{---} & 1.6 & 1.9 & 2.1 & 1.9 & \multirow{3}{*}{---} \\
\hline & 500 & 1.4 & 1.6 & 1.7 & 1.5 & & 1.5 & 1.7 & 1.8 & 1.7 & \\
\hline \multicolumn{2}{|c|}{ Overall mean } & 1.4 & 1.7 & 1.8 & --- & & 1.6 & 1.8 & 1.98 & --- & \\
\hline \multirow{4}{*}{\multicolumn{3}{|c|}{$\begin{array}{l}\text { L.S.D. at } 5 \% \text { for: } \\
\text { Treatments }(\mathrm{T})=0.10 \\
\text { Nitrogen rates }(\mathrm{N})=0.06 \\
\text { Cuttings }(\mathrm{C}) \quad=0.07\end{array}$}} & \multicolumn{4}{|c|}{$\mathrm{T} \times \mathrm{N}=\mathrm{ns}$} & & & $\mathrm{xN}$ & $=0.03$ & \\
\hline & & & $\mathrm{T} \mathrm{x}$ & & & & $\mathrm{T}=0$ & & $\mathrm{x} \mathrm{C}$ & $=0.04$ & \\
\hline & & & $\mathrm{Nx}$ & 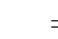 & & & $\mathrm{N}=0$ & & $x \mathrm{C}$ & $=0.02$ & \\
\hline & & & $\mathrm{T} x$ & $x C=$ & & & $\mathrm{C}=0$ & & $\mathrm{x} \mathrm{Nx}$ & $=0.05$ & \\
\hline
\end{tabular}

Data of field experiments shown in Tables 5 and 6 indicate that using any of the tested anti-transpirants applied as foliar spray treatments at two nitrogen rates caused a significant protection against downy mildew infection during the two seasons. It is evident that between the anti-transpirants tested, potassium silicate showed superiority in reducing downy mildew infection through the two successive seasons when applied on plants given recommended rate of nitrogen (400 kg/fed.). The corresponding mean values of reduction in downy mildew incidence were 70.2 and $73.9 \%$ and disease severity, being 19.9 and $16.7 \%$, through 2015 and 2016 growing seasons, respectively. It is worthy to note that treatment with fungicide Stone $50 \%$ gave the highest protection against downy mildew of basil when applied on plants received the recommended rate of nitrogen fertilizer $(400 \mathrm{~kg} / \mathrm{fed}$.). Green miracle came in the second rank and showed moderate plant protection against downy mildew infection followed by silicon dioxide. The least effective treatment was aluminum silicate. Generally, the severity of downy mildew was increased when nitrogen was added at a rate above the recommended and showed the maximum increase in the second plant cut. 
Table 5. Reduction in percentage of disease incidence due to different antitranspirant treatments applied as foliar spray at two nitrogen rates against downy mildew of basil for three plant cuts during two growing seasons 2015 and 2016 under field conditions

\begin{tabular}{|c|c|c|c|c|c|c|c|c|c|c|c|}
\hline \multirow[b]{2}{*}{ Treatment } & \multirow[b]{2}{*}{$\begin{array}{l}\text { N. rates } \\
\text { (kg/fed.) }\end{array}$} & \multicolumn{5}{|c|}{ Season 2015} & \multicolumn{5}{|c|}{ Season 2016} \\
\hline & & $\begin{array}{l}1^{\text {st }} \\
\text { cut }\end{array}$ & $\begin{array}{l}2^{\text {nd }} \\
\text { cut }\end{array}$ & $\begin{array}{l}3^{\text {rd }} \\
\text { cut }\end{array}$ & Mean & $\begin{array}{l}\text { Overall } \\
\text { mean }\end{array}$ & $\begin{array}{l}1^{\text {st }} \\
\text { cut }\end{array}$ & $\begin{array}{l}2^{\text {nd }} \\
\text { cut }\end{array}$ & $\begin{array}{l}3^{\text {rd }} \\
\text { cut }\end{array}$ & Mean & $\begin{array}{l}\text { Overall } \\
\text { mean }\end{array}$ \\
\hline \multirow{2}{*}{ Green miracle } & 400 & 53.5 & 42.1 & 48.3 & 48.0 & \multirow{3}{*}{46.5} & 55.3 & 43.7 & 50.8 & 49.9 & \multirow{3}{*}{48.1} \\
\hline & 500 & 51.7 & 40.9 & 42.0 & 45.0 & & 52.3 & 42.0 & 44.7 & 46.3 & \\
\hline \multicolumn{2}{|c|}{ Mean } & 52.6 & 41.5 & 45.2 & --- & & 53.8 & 43.0 & 47.8 & --- & \\
\hline \multirow{2}{*}{$\begin{array}{c}\text { Aluminum } \\
\text { silicate } \\
\left(\mathrm{Al}_{2} \mathrm{SiO}_{5}\right) \\
\text { (Kaolin) }\end{array}$} & 400 & 21.4 & 12.7 & 16.3 & 16.7 & \multirow{3}{*}{15.8} & 22.9 & 13.3 & 17.5 & 17.9 & \multirow{3}{*}{17.1} \\
\hline & 500 & 19.5 & 10.0 & 15.3 & 14.8 & & 21.1 & 11.5 & 16.6 & 16.4 & \\
\hline \multicolumn{2}{|c|}{ Mean } & 20.5 & 11.4 & 15.3 & --- & & 22.0 & 12.4 & 17.0 & --- & \\
\hline \multirow{2}{*}{$\begin{array}{c}\text { Potassium } \\
\text { silicate } \\
\left(\mathrm{K}_{2} \mathrm{O}_{3} \mathrm{Si}\right)\end{array}$} & 400 & 78.3 & 63.0 & 69.0 & 70.2 & \multirow{3}{*}{69.5} & 82.9 & 65.7 & 73.1 & 73.9 & \multirow{3}{*}{72.6} \\
\hline & 500 & 78.0 & 60.2 & 68.0 & 68.7 & & 81.2 & 62.9 & 70.0 & 71.4 & \\
\hline \multicolumn{2}{|c|}{ Mean } & 78.1 & 61.6 & 68.5 & --- & & 82.1 & 64.3 & 71.6 & --- & \\
\hline \multirow{2}{*}{$\begin{array}{c}\text { Silicon dioxide } \\
\left(\mathrm{SiO}_{2}\right)\end{array}$} & 400 & 29.9 & 19.5 & 26.0 & 25.1 & \multirow{3}{*}{24.1} & 31.0 & 21.9 & 28.9 & 27.3 & \multirow{3}{*}{26.1} \\
\hline & 500 & 28.0 & 18.3 & 22.0 & 23.0 & & 30.7 & 20.0 & 24.1 & 24.9 & \\
\hline \multicolumn{2}{|c|}{ Mean } & 29.0 & 19.0 & 24.0 & --- & & 30.8 & 21.0 & 26.5 & --- & \\
\hline \multirow{2}{*}{ Stone $50 \%$} & 400 & 97.8 & 89.3 & 91.0 & 92.8 & \multirow{3}{*}{91.7} & 100.0 & 92.5 & 96.0 & 96.2 & \multirow{3}{*}{94.7} \\
\hline & 500 & 95.1 & 85.5 & 91.3 & 90.5 & & 98.7 & 87.3 & 93.8 & 93.3 & \\
\hline \multicolumn{2}{|c|}{ Mean } & 96.4 & 87.4 & 91.2 & --- & & 99.3 & 89.9 & 94.9 & --- & \\
\hline \multirow{2}{*}{ Control } & 400 & 0.0 & 0.0 & 0.0 & 0.0 & \multirow{3}{*}{0.0} & 0.0 & 0.0 & 0.0 & 0.0 & \multirow{3}{*}{0.0} \\
\hline & 500 & 0.0 & 0.0 & 0.0 & 0.0 & & 0.0 & 0.0 & 0.0 & 0.0 & \\
\hline \multicolumn{2}{|c|}{ Mean } & 0.0 & 0.0 & 0.0 & --- & & 0.0 & 0.0 & 0.0 & --- & \\
\hline Mean of & 400 & 46.8 & 37.8 & 41.8 & 42.1 & \multirow{3}{*}{--- } & 48.7 & 39.5 & 44.4 & 44.2 & \multirow{3}{*}{---} \\
\hline nitrogen rates & 500 & 45.4 & 35.9 & 39.9 & 40.4 & & 47.3 & 37.3 & 41.5 & 42.0 & \\
\hline Overall $\mathrm{r}$ & nean & 46.1 & 36.8 & 40.8 & --- & & 48.0 & 38.4 & 43.0 & --- & \\
\hline L.S.D. at $5 \%$ & for: & & & $=0.26$ & & & & & $\mathrm{~T} \times \mathrm{N}$ & $=0.2$ & \\
\hline Treatments ( $\mathrm{T}$ & $=0.52$ & & & $=0.32$ & & & $\mathrm{~T}=0$. & & $\mathrm{T} \times \mathrm{C}$ & $=0.3$ & \\
\hline Nitrogen rates & $(\mathrm{N})=0.11$ & & $\mathrm{C}$ & $=0.18$ & & & $\mathrm{~N}=0$ & & $\mathrm{~N} \times \mathrm{C}$ & $=0.1$ & \\
\hline Cuttings ( C ) & $=0.13$ & & $\mathrm{~N} \times \mathrm{C}$ & $=0.45$ & & & $\mathrm{C}=0$. & & $\mathrm{T} \times \mathrm{N} x$ & $\mathrm{C}=0.4$ & \\
\hline
\end{tabular}


Table 6. Evaluation of different anti-transpirant treatments applied as foliar spray at two nitrogen rates on disease severity of basil downy mildew for three plant cuts during two growing seasons 2015 and 2016 under field conditions

\begin{tabular}{|c|c|c|c|c|c|c|c|c|c|c|c|}
\hline \multirow{3}{*}{ Treatment } & \multirow{3}{*}{$\begin{array}{l}\text { N. rates } \\
(\mathrm{kg} / \mathrm{fed})\end{array}$} & \multicolumn{10}{|c|}{ Disease severity \% } \\
\hline & & \multicolumn{5}{|c|}{ Season 2015} & \multicolumn{5}{|c|}{ Season 2016} \\
\hline & & $\begin{array}{l}1^{\mathrm{st}} \\
\text { cut }\end{array}$ & $\begin{array}{l}2^{\text {nd }} \\
\text { cut }\end{array}$ & $\begin{array}{l}3^{\text {rd }} \\
\text { cut }\end{array}$ & Mean & $\begin{array}{r}\text { Overall } \\
\text { mean }\end{array}$ & $\begin{array}{l}1^{\text {st }} \\
\text { cut }\end{array}$ & $\begin{array}{l}2^{\text {nd }} \\
\text { cut }\end{array}$ & $\begin{array}{l}3^{\text {rd }} \\
\text { cut }\end{array}$ & Mean & $\begin{array}{c}\text { Overall } \\
\text { mean }\end{array}$ \\
\hline \multirow{2}{*}{ Green miracle } & 400 & 23.6 & 38.4 & 25.0 & 29.0 & \multirow{3}{*}{29.8} & 21.6 & 36.5 & 22.1 & 26.7 & \multirow{3}{*}{28.1} \\
\hline & 500 & 23.9 & 38.8 & 28.9 & 30.5 & & 23.5 & 37.2 & 27.8 & 29.5 & \\
\hline \multicolumn{2}{|l|}{ Mean } & 23.7 & 38.6 & 26.9 & $\begin{array}{ll}-- \\
\end{array}$ & & 22.6 & 36.8 & 24.9 & --- & \\
\hline \multirow{2}{*}{$\begin{array}{l}\text { Aluminum silicate } \\
\left(\mathrm{Al}_{2} \mathrm{SiO}_{5}\right)(\text { Kaolin })\end{array}$} & 400 & 29.1 & 41.1 & 31.0 & 33.7 & \multirow{3}{*}{35.1} & 27.3 & 40.2 & 30.5 & 32.7 & \multirow{3}{*}{34.2} \\
\hline & 500 & 29.5 & 42.9 & 37.0 & 36.5 & & 28.5 & 42.0 & 36.4 & 35.6 & \\
\hline \multicolumn{2}{|l|}{ Mean } & 29.3 & 42.0 & 34.0 & --- & & 27.9 & 41.1 & 33.5 & --- & \\
\hline \multirow{2}{*}{$\begin{array}{c}\text { Potassium silicate } \\
\qquad\left(\mathrm{K}_{2} \mathrm{O}_{3} \mathrm{Si}\right)\end{array}$} & 400 & 9.9 & 28.0 & 21.7 & 19.9 & \multirow{3}{*}{20.4} & 6.5 & 25.7 & 18.0 & 16.7 & \multirow{3}{*}{17.4} \\
\hline & 500 & 11.3 & 28.9 & 22.5 & 20.9 & & 8.0 & 26.0 & 20.5 & 18.2 & \\
\hline \multicolumn{2}{|l|}{ Mean } & 10.6 & 28.4 & 22.1 & --- & & 7.2 & 25.8 & 19.3 & --- & \\
\hline \multirow{2}{*}{ Silicon dioxide $\left(\mathrm{SiO}_{2}\right)$} & 400 & 22.9 & 38.6 & 30.1 & 30.5 & \multirow{3}{*}{31.4} & 20.6 & 37.9 & 28.0 & 28.8 & \multirow{3}{*}{29.3} \\
\hline & 500 & 25.2 & 39.0 & 32.4 & 32.2 & & 21.9 & 38.9 & 28.4 & 29.8 & \\
\hline \multicolumn{2}{|l|}{ Mean } & 24.0 & 38.8 & 31.2 & $\begin{array}{ll}-- \\
\end{array}$ & & 21.3 & 38.4 & 28.2 & --- & \\
\hline \multirow{2}{*}{ Stone $50 \%$} & 400 & 1.2 & 8.1 & 2.2 & 3.8 & \multirow{3}{*}{5.3} & 0.0 & 7.0 & 1.6 & 2.9 & \multirow{3}{*}{3.8} \\
\hline & 500 & 2.5 & 13.9 & 3.9 & 6.8 & & 1.2 & 10.7 & 2.0 & 4.6 & \\
\hline \multicolumn{2}{|l|}{ Mean } & 1.9 & 11.1 & 3.0 & --- & & 0.6 & 8.9 & 1.8 & --- & \\
\hline \multirow{2}{*}{ Control } & 400 & 48.6 & 63.1 & 54.3 & 55.3 & \multirow{3}{*}{57.5} & 50.9 & 66.5 & 55.2 & 57.5 & \multirow{3}{*}{60.3} \\
\hline & 500 & 51.7 & 70.8 & 56.5 & 59.7 & & 55.2 & 74.3 & 59.7 & 63.1 & \\
\hline Mean & & 50.2 & 66.9 & 55.4 & --- & & 53.1 & 70.4 & 57.4 & --- & \\
\hline & 400 & 22.5 & 36.2 & 27.4 & 28.7 & \multirow{3}{*}{--} & 21.2 & 35.6 & 25.9 & 27.6 & \\
\hline Mean of nitrogen rates & 500 & 24.0 & 39.0 & 30.2 & 31.1 & & 23.1 & 38.2 & 29.1 & 30.1 & --- \\
\hline Overall mean & & 23.3 & 37.6 & 28.8 & $\begin{array}{ll}--- \\
\end{array}$ & & 22.1 & 36.9 & 27.5 & --- & \\
\hline L.S.D. at $5 \%$ for: & & $\mathrm{N}$ & $=1.3$ & & & & & & $\mathrm{~T} \times \mathrm{N}$ & & .28 \\
\hline Treatments $(\mathrm{T})$ & & $\mathrm{C}$ & $=1$. & & & & $\mathrm{T}=$ & & $\mathrm{T} \times \mathrm{C}$ & & 0.34 \\
\hline Nitrogen rates $(N)=0$ & & x C & $=0.9$ & & & & $\mathrm{~N}=0$ & & $\mathrm{~N} \times \mathrm{C}$ & & 0.19 \\
\hline Cuttings ( C ) & & $\mathrm{x} \mathrm{N} \times \mathrm{C}$ & $C=2$. & & & & $\mathrm{C}=0$ & & $\mathrm{~T} \times \mathrm{N}$ & $\mathrm{J} \times \mathrm{C}=$ & 0.48 \\
\hline
\end{tabular}

The tested anti-transpirants significantly affected the growth of basil plants by increasing the fresh and dry weights of herb than the untreated control (Tables 7 and 8 ). It is clear that maximum mean values of fresh and dry weights of herb in ton per fed. for the three cuts were obtained due to using of the potassium silicate treatment applied at the two rates of nitrogen fertilizer. The corresponding mean values of fresh and dry weights in the first season (2015) were $7.9 \& 1.7$ ton/fed. respectively, when the plants received the recommended rate of nitrogen fertilizer $(400 \mathrm{~kg} / \mathrm{fed}$.), being $8.6 \& 1.6$ ton/fed. when plants received high rate of nitrogen fertilizer (500 $\mathrm{kg} / \mathrm{fed}$.), followed by green miracle and silicon dioxide treatments. A similar trend was obtained in the second season (2016). The lowest values of these plant growth parameters were found in kaolin (Aluminum silicate) treatment. 
Table 7. Effect of anti-transpirant treatments applied as foliar spray at two nitrogen rates on fresh weight of herb (ton/fed.) for three cuts of basil plants naturally infected by downy mildew during two growing seasons 2015 and 2016 under field conditions

\begin{tabular}{|c|c|c|c|c|c|c|c|c|c|c|c|}
\hline \multirow{3}{*}{ Treatment } & \multirow{3}{*}{ 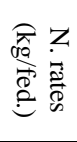 } & \multicolumn{10}{|c|}{ Fresh weight of herb (ton/fed.) } \\
\hline & & \multicolumn{5}{|c|}{ Season 2015} & \multicolumn{5}{|c|}{ Season 2016} \\
\hline & & $\begin{array}{l}1^{\mathrm{st}} \\
\text { cut }\end{array}$ & $\begin{array}{l}2^{\text {nd }} \\
\text { cut }\end{array}$ & $\begin{array}{l}3^{\text {rd }} \\
\text { cut }\end{array}$ & Mean & $\begin{array}{l}\text { Overall } \\
\text { mean }\end{array}$ & $\begin{array}{l}1^{\text {st }} \\
\text { cut }\end{array}$ & $\begin{array}{l}2^{\text {nd }} \\
\text { cut }\end{array}$ & $\begin{array}{l}3^{\text {rd }} \\
\text { cut }\end{array}$ & Mean & $\begin{array}{l}\text { Overall } \\
\text { mean }\end{array}$ \\
\hline \multirow{2}{*}{ Green miracle } & 400 & 4.1 & 5.3 & 6.0 & 5.1 & \multirow{3}{*}{5.3} & 4.7 & 5.7 & 6.6 & 5.7 & \multirow{3}{*}{5.8} \\
\hline & 500 & 4.3 & 5.5 & 6.6 & 5.5 & & 5.0 & 6.0 & 7.0 & 6.0 & \\
\hline \multicolumn{2}{|l|}{ Mean } & 4.2 & 5.4 & 6.3 & --- & & 4.8 & 5.8 & 6.8 & --- & \\
\hline \multirow{2}{*}{$\begin{array}{l}\text { Aluminum silicate } \\
\left(\mathrm{Al}_{2} \mathrm{SiO}_{5}\right)(\text { Kaolin })\end{array}$} & 400 & 3.4 & 4.2 & 4.9 & 4.2 & \multirow{3}{*}{4.4} & 3.9 & 4.4 & 5.5 & 4.6 & \multirow{3}{*}{4.8} \\
\hline & 500 & 3.7 & 4.6 & 5.4 & 4.6 & & 4.2 & 5.0 & 6.0 & 5.1 & \\
\hline \multicolumn{2}{|l|}{ Mean } & 3.5 & 4.4 & 5.2 & --- & & 4.0 & 4.7 & 5.8 & --- & \\
\hline \multirow[t]{2}{*}{\begin{tabular}{|l|} 
Potassium silicate \\
\end{tabular}} & 400 & 6.10 & 8.1 & 9.6 & 7.9 & \multirow{3}{*}{8.3} & 6.6 & 9.8 & 10.5 & 9.0 & \multirow{3}{*}{9.4} \\
\hline & 500 & 6.8 & 8.8 & 10.1 & 8.6 & & 7.1 & 10.8 & 11.3 & 9.7 & \\
\hline \multicolumn{2}{|l|}{ Mean } & 6.5 & 8.9 & 9.8 & --- & & 6.8 & 10.3 & 10.9 & --- & \\
\hline \multirow[t]{2}{*}{ Silicon dioxide } & 400 & 3.9 & 4.7 & 5.7 & 4.8 & \multirow{3}{*}{4.9} & 4.1 & 5.1 & 6.0 & 5.1 & \multirow{3}{*}{5.3} \\
\hline & 500 & 4.2 & 4.9 & 6.0 & 5.0 & & 4.6 & 5.3 & 6.5 & 5.5 & \\
\hline \multicolumn{2}{|l|}{ Mean } & 4.1 & 4.8 & 5.8 & --- & & 4.3 & 5.2 & 6.3 & --- & \\
\hline \multirow{2}{*}{ Stone $50 \%$} & 400 & 8.1 & 10.2 & 12.4 & 10.2 & \multirow{3}{*}{10.5} & 8.9 & 11.0 & 13.4 & 11.1 & \multirow{3}{*}{11.3} \\
\hline & 500 & 8.7 & 10.6 & 12.9 & 10.7 & & 9.5 & 11.6 & 13.6 & 11.6 & \\
\hline \multicolumn{2}{|l|}{ Mean } & 8.4 & 10.4 & 12.7 & $\begin{array}{ll}-- \\
-1\end{array}$ & & 9.2 & 11.3 & 13.5 & $\begin{array}{ll}-- \\
-\end{array}$ & \\
\hline \multirow{2}{*}{ Control } & 400 & 3.2 & 3.7 & 4.3 & 3.8 & \multirow{3}{*}{3.9} & 2.9 & 3.4 & 4.1 & 3.5 & \multirow{3}{*}{3.7} \\
\hline & 500 & 3.4 & 3.9 & 4.7 & 4.0 & & 3.3 & 3.8 & 4.4 & 3.8 & \\
\hline Mean & & 3.3 & 3.8 & 4.5 & --- & & 3.1 & 3.6 & 4.3 & $\begin{array}{ll}-- \\
\end{array}$ & \\
\hline Mean of nitrogen & 400 & 4.8 & 6.0 & 7.2 & 6.0 & & 5.1 & 6.6 & 7.7 & 6.5 & \\
\hline rates & 500 & 5.2 & 6.4 & 7.6 & 6.4 & --- & 5.6 & 7.1 & 8.1 & 6.9 & --- \\
\hline Overall mea & & 5.0 & 6.2 & 7.4 & --- & & 5.4 & 6.8 & 7.9 & --- & \\
\hline L.S.D. at $5 \%$ for: & & & & $=\mathrm{ns}$ & & & & & $\mathrm{T} \times \mathrm{N}$ & & ns \\
\hline Treatments $(\mathrm{T})$ & $=0.22$ & & & $=0.39$ & & & $\mathrm{~T}=\mathrm{C}$ & & $\mathrm{T} \times \mathrm{C}$ & & 0.38 \\
\hline Nitrogen rates $(\mathrm{N})$ & $=0.13$ & & & $=\mathrm{ns}$ & & & $\mathrm{N}=($ & & $\mathrm{N} \times \mathrm{C}$ & $=$ & ns \\
\hline Cuttings ( C ) & $=0.16$ & & $\mathrm{~N} \times \mathrm{C}$ & $=\mathrm{ns}$ & & & $\mathrm{C}=\mathrm{C}$ & & $\mathrm{T} \times \mathrm{N}_{x}$ & $\mathrm{xC}=$ & ns \\
\hline
\end{tabular}

Egypt. J. Phytopathol., Vol. 45, No. 1 (2017) 
Table 8. Effect of anti-transpirant treatments applied as foliar spray at two nitrogen rates dry weight of herb (ton/fed.) for three cuts of basil plants naturally infected by downy mildew during two growing seasons 2015 and 2016 under field conditions

\begin{tabular}{|c|c|c|c|c|c|c|c|c|c|c|c|}
\hline \multirow{3}{*}{ Treatment } & \multirow{3}{*}{ 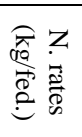 } & \multicolumn{10}{|c|}{ Herb dry weight (ton/fed.) } \\
\hline & & \multicolumn{5}{|c|}{ Season 2015} & \multicolumn{5}{|c|}{ Season 2016} \\
\hline & & $\begin{array}{l}1^{\text {st }} \\
\text { cut }\end{array}$ & $\begin{array}{l}2^{\text {nd }} \\
\text { cut }\end{array}$ & $\begin{array}{l}3^{\text {rd }} \\
\text { cut }\end{array}$ & Mean & $\begin{array}{l}\text { Pverall } \\
\text { mean }\end{array}$ & $\begin{array}{l}1^{\text {st }} \\
\text { cut }\end{array}$ & $\begin{array}{l}2^{\text {nd }} \\
\text { cut }\end{array}$ & $\begin{array}{l}3^{\text {rd }} \\
\text { cut }\end{array}$ & Mean & $\begin{array}{c}\text { Overall } \\
\text { mean }\end{array}$ \\
\hline \multirow{2}{*}{ Green miracle } & 400 & 1.0 & 1.1 & 1.3 & 1.1 & \multirow{3}{*}{1.1} & 1.1 & 1.3 & 1.6 & 1.3 & \multirow{3}{*}{1.3} \\
\hline & 500 & 0.9 & 1.0 & 1.2 & 1.0 & & 1.1 & 1.2 & 1.4 & 1.2 & \\
\hline \multicolumn{2}{|l|}{ Mean } & 0.9 & 1.0 & 1.3 & --- & & 1.1 & 1.2 & 1.5 & --- & \\
\hline \multirow{2}{*}{$\begin{array}{l}\text { Aluminum silicate } \\
\left(\mathrm{Al}_{2} \mathrm{SiO}_{5}\right) \text { (Kaolin) }\end{array}$} & 400 & 0.8 & 1.0 & 1.1 & 1.0 & \multirow{3}{*}{0.9} & 0.8 & 1.1 & 1.4 & 1.1 & \multirow{3}{*}{1.0} \\
\hline & 500 & 0.7 & 0.8 & 0.9 & 0.8 & & 0.7 & 0.9 & 1.0 & 0.9 & \\
\hline \multicolumn{2}{|l|}{ Mean } & 0.7 & 0.9 & 1.0 & --- & & 0.7 & 1.0 & 1.2 & --- & \\
\hline \multirow{2}{*}{$\begin{array}{c}\text { Potassium silicate } \\
\left(\mathrm{K}_{2} \mathrm{O}_{3} \mathrm{Si}\right)\end{array}$} & 400 & 1.5 & 1.7 & 1.9 & 1.7 & \multirow{3}{*}{1.6} & 1.6 & 2.0 & 2.2 & 2.0 & \multirow{3}{*}{1.8} \\
\hline & 500 & 1.4 & 1.6 & 1.7 & 1.6 & & 1.4 & 1.7 & 2.0 & 1.7 & \\
\hline \multicolumn{2}{|l|}{ Mean } & 1.4 & 1.6 & 1.8 & --- & & 1.5 & 1.9 & 2.10 & --- & \\
\hline \multirow{2}{*}{$\begin{array}{l}\text { Silicon dioxide } \\
\quad\left(\mathrm{SiO}_{2}\right)\end{array}$} & 400 & 0.9 & 1.0 & $\begin{array}{c}1.2 \\
3\end{array}$ & 1.0 & \multirow{3}{*}{1.0} & 0.9 & 1.2 & 1.5 & 1.2 & \multirow{3}{*}{1.1} \\
\hline & 500 & 0.8 & 0.9 & 1.1 & 0.9 & & 0.9 & 1.0 & 1.20 & 1.03 & \\
\hline \multicolumn{2}{|l|}{ Mean } & 0.8 & 0.9 & 1.2 & --- & & 0.9 & 1.1 & 1.4 & --- & \\
\hline \multirow{2}{*}{ Stone $50 \%$} & 400 & 2.0 & 2.3 & 2.5 & 2.3 & \multirow{3}{*}{2.2} & 2.2 & 2.4 & 2.7 & 2.4 & \multirow{3}{*}{2.4} \\
\hline & 500 & 1.8 & 2.1 & 2.3 & 2.0 & & 2.0 & 2.3 & 2.5 & 2.3 & \\
\hline \multicolumn{2}{|l|}{ Mean } & 1.9 & 2.2 & 2.4 & --- & & 2.1 & 2.4 & 2.6 & --- & \\
\hline \multirow{2}{*}{ Control } & 400 & 0.8 & 1.0 & 1.1 & 1.0 & \multirow{3}{*}{0.9} & 0.8 & 0.9 & 1.1 & 0.9 & \multirow{3}{*}{0.8} \\
\hline & 500 & 0.7 & 0.8 & 0.8 & 0.7 & & 0.6 & 0.7 & 0.7 & 0.7 & \\
\hline \multicolumn{2}{|l|}{ Mean } & 0.7 & 0.9 & 0.9 & --- & & 0.7 & 0.8 & 0.9 & --- & \\
\hline Mean of nitrogen & 400 & 1.2 & 1.4 & 1.5 & 1.3 & & 1.2 & 1.5 & 1.8 & 1.5 & \\
\hline rates & 500 & 1.0 & 1.2 & 1.3 & 1.2 & & 1.1 & 1.3 & 1.5 & 1.3 & \\
\hline Overall mea & & 1.1 & 1.3 & 1.4 & --- & & $\begin{array}{c}1.1 \\
8\end{array}$ & 1.4 & 1.6 & --- & \\
\hline L.S.D. at $5 \%$ for: & & & & $=0.03$ & & & & & $\mathrm{~T} \times \mathrm{N}$ & $=0.0$ & \\
\hline Treatments $(\mathrm{T})$ & $=0.02$ & & & $=0.04$ & & & $\mathrm{~T}=\mathrm{C}$ & & $\mathrm{T} \times \mathrm{C}$ & $=0.04$ & \\
\hline Nitrogen rates $(\mathrm{N})$ & $=0.01$ & & & $=0.02$ & & & $\mathrm{~N}=$ & & $\mathrm{N} \times \mathrm{C}$ & $=0.02$ & \\
\hline Cuttings ( C ) & $=0.01$ & & $\mathrm{~N} \times \mathrm{C}$ & $=0.05$ & & & $\mathrm{C}=\mathrm{C}$ & & $\mathrm{T} \times \mathrm{N} x$ & $C=0.05$ & \\
\hline
\end{tabular}

The obtained data Tables, 9 and 10 show that spraying any of mineral salts tested, i.e. di-potassium phosphate, mono-potassium phosphate and potassium carbonate significantly affected the disease compared to the untreated control. Di-potassium phosphate applied weekly provided the most efficacious control of basil downy mildew in both seasons at the two rates of nitrogen fertilizer. The reduction in disease incidence reached 73.6 and $77.8 \%$ on plants given the recommended rate of nitrogen fertilizer (400 kg/fed.) through the two growing seasons, respectively. While, it reached 72.2 and $75.9 \%$ at the high rate of nitrogen fertilizer $(500 \mathrm{~kg} / \mathrm{fed}$.), respectively. On the other hand, the disease severity value was significantly lower compared to the other treatments which recorded 16.2 and $13.8 \%$ at the recommended rate of nitrogen and 19.5 and $17.5 \%$ at the high rate of nitrogen fertilizer in both experimental seasons, respectively. Moderate disease suppression was provided with potassium carbonate. Meanwhile mono-potassium phosphate was the lowest efficient salt in controlling basil downy mildew. In general, the fungicide Stone $50 \%$ was more effective than the other treatments in minimizing the disease incidence and severity in both seasons. 
Table 9. Reduction in percentage of disease incidence due to different mineral salts applied as foliar spray at two nitrogen rates against downy mildew of basil for three plant cuts during two growing seasons 2015 and 2016 under field conditions

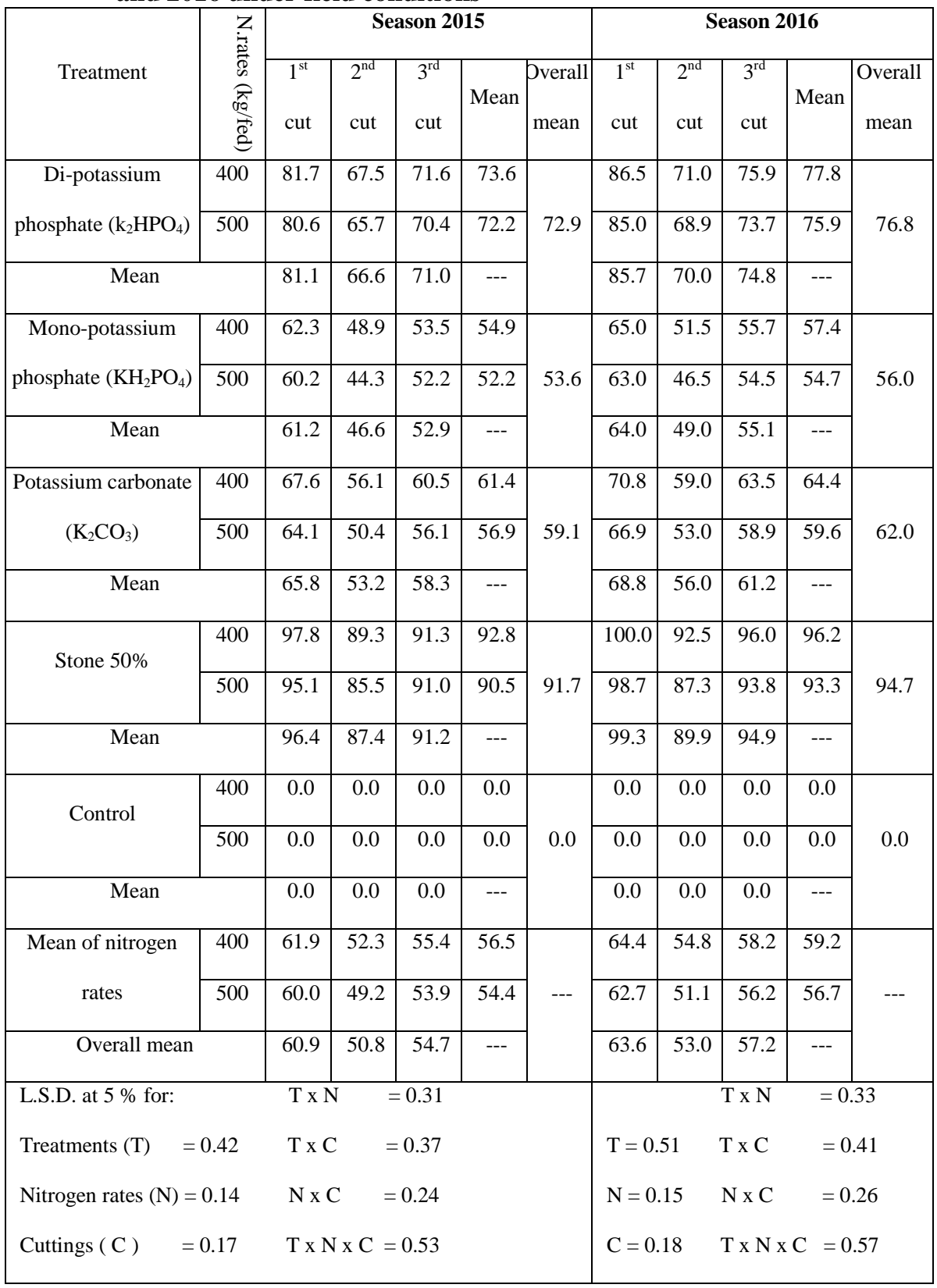


Table 10. Evaluation of different mineral salts applied as foliar spray at two nitrogen rates on disease severity of basil downy mildew for three plant cuts during two growing seasons 2015 and 2016 under field conditions

\begin{tabular}{|c|c|c|c|c|c|c|c|c|c|c|c|}
\hline \multirow{3}{*}{ Treatment } & \multirow{3}{*}{ 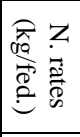 } & \multicolumn{10}{|c|}{ Disease severity \% } \\
\hline & & \multicolumn{5}{|c|}{ Season 2015} & \multicolumn{5}{|c|}{ Season 2016} \\
\hline & & $\begin{array}{l}1^{\mathrm{st}} \\
\text { cut }\end{array}$ & $\begin{array}{l}2^{\text {nd }} \\
\text { cut }\end{array}$ & $\begin{array}{l}3^{\text {rd }} \\
\text { cut }\end{array}$ & Mean & $\begin{array}{l}\text { Pverall } \\
\text { mean }\end{array}$ & $\begin{array}{l}1^{\text {st }} \\
\text { cut }\end{array}$ & $\begin{array}{l}2^{\text {nd }} \\
\text { cut }\end{array}$ & $\begin{array}{l}3^{\text {rd }} \\
\text { cut }\end{array}$ & Mean & $\begin{array}{c}\text { Overall } \\
\text { mean }\end{array}$ \\
\hline \multirow{2}{*}{$\begin{array}{c}\text { Di-potassium } \\
\text { phosphate }\left(\mathrm{k}_{2} \mathrm{HPO}_{4}\right)\end{array}$} & 400 & 6.6 & 27.4 & 14.7 & 16.2 & \multirow{3}{*}{17.9} & 5.5 & 24.5 & 11.5 & 13.8 & \multirow{3}{*}{15.7} \\
\hline & 500 & 8.8 & 28.3 & 21.5 & 19.5 & & 6.9 & 26.0 & 19.5 & 17.5 & \\
\hline \multicolumn{2}{|l|}{ Mean } & 7.7 & 27.8 & 18.1 & --- & & 6.2 & 25.2 & 15.5 & --- & \\
\hline \multirow{2}{*}{$\begin{array}{c}\text { Mono-potassium } \\
\text { phosphate } \\
\left(\mathrm{KH}_{2} \mathrm{PO}_{4}\right) \\
\end{array}$} & 400 & 15.6 & 33.3 & 20.9 & 23.3 & \multirow{3}{*}{24.5} & 13.5 & 32.0 & 18.5 & 21.4 & \multirow{3}{*}{22.8} \\
\hline & 500 & 15.7 & 34.9 & 26.8 & 25. & & 13.9 & 33.0 & 25.7 & 24.2 & \\
\hline \multicolumn{2}{|l|}{ Mean } & 15.6 & 34.1 & 23.8 & --- & & 13.7 & 32.5 & 22.1 & --- & \\
\hline \multirow{2}{*}{$\begin{array}{c}\text { Potassium carbonate } \\
\left(\mathrm{K}_{2} \mathrm{CO}_{3}\right)\end{array}$} & 400 & 11.1 & 32.8 & 24.9 & 22.9 & \multirow{3}{*}{24.8} & 9.0 & 30.6 & 22.7 & 20.8 & \multirow{3}{*}{22.8} \\
\hline & 500 & 14.7 & 36.9 & 28.6 & 26.7 & & 12.6 & 35.9 & 26.3 & 24.9 & \\
\hline \multicolumn{2}{|l|}{ Mean } & 12.9 & 34.8 & 26.7 & --- & & 10.8 & 33.2 & 24.5 & --- & \\
\hline \multirow{2}{*}{ Stone $50 \%$} & 400 & 1.2 & 8.1 & 2.2 & 3.8 & \multirow{3}{*}{5.3} & 0.0 & 7.0 & 1.6 & 2.9 & \multirow{3}{*}{3.8} \\
\hline & 500 & 2.5 & 13.9 & 3.9 & 6.8 & & 1.3 & 10.7 & 2.0 & 4.6 & \\
\hline \multicolumn{2}{|l|}{ Mean } & 1.9 & 11.0 & 3.1 & --- & & 0.6 & 8.9 & 1.8 & --- & \\
\hline \multirow{2}{*}{ Control } & 400 & 48.6 & 63.1 & 54.3 & 55.3 & \multirow{3}{*}{57.5} & 50.9 & 66.5 & 55.2 & 57.54 & \multirow{3}{*}{60.3} \\
\hline & 500 & 51.7 & 70.8 & 56.5 & 59.7 & & 55.2 & 74.3 & 59.7 & 63.1 & \\
\hline \multicolumn{2}{|l|}{ Mean } & 50.2 & 66.9 & 55.4 & --- & & 53.1 & 70.4 & 57.4 & --- & \\
\hline \multirow{2}{*}{$\begin{array}{c}\text { Mean of nitrogen } \\
\text { rates }\end{array}$} & 400 & 16.6 & 32.9 & 23.4 & 24.3 & \multirow{3}{*}{---} & 15.8 & 32.1 & 21.9 & 23.3 & \multirow{3}{*}{--- } \\
\hline & 500 & 18.7 & 36.9 & 27.44 & 27.8 & & 18.0 & 36.0 & 26.6 & 26.9 & \\
\hline \multicolumn{2}{|l|}{ Overall mean } & 17.7 & 34.9 & 25.42 & --- & & 16.9 & 34.5 & 24.3 & --- & \\
\hline \multirow{4}{*}{\multicolumn{2}{|c|}{ 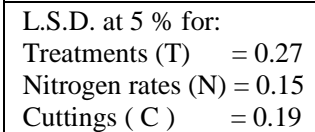 }} & \multirow{2}{*}{\multicolumn{2}{|c|}{$\begin{array}{l}\mathrm{T} \times \mathrm{N} \\
\mathrm{T} \times \mathrm{C}\end{array}$}} & $=0.34$ & & & & & $\mathrm{~T} \times \mathrm{N}$ & $=0$. & \\
\hline & & & & $=0.42$ & & & $\mathrm{~T}=0$ & & $\mathrm{~T} \times \mathrm{C}$ & $=0.4$ & \\
\hline & & & & $=0.26$ & & & $\mathrm{~N}=\mathrm{C}$ & & $\mathrm{N} \times \mathrm{C}$ & $=0.2$ & \\
\hline & & & $\mathrm{N} \times \mathrm{C}$ & $=0.59$ & & & $\mathrm{C}=$ & & $\mathrm{T} \times \mathrm{Nx}$ & $\mathrm{C}=0.6$ & \\
\hline
\end{tabular}

The efficacy of all tested mineral salts in disease control was reflected on the plant yield, causing significant increase in the fresh and dry weights of herb (Tables 11 and 12). The pronounced increase in this respect was observed with di-potassium phosphate treatment which resulted in the highest mean values of herb fresh weight (8.6 ton/fed.) as well as dry weight of herb (1.8 ton/fed.) when nitrogen fertilizer was applied at the recommended rate (400 kg/fed.) and 9.0 and $1.6 \mathrm{ton} / \mathrm{fed}$., respectively when nitrogen fertilizer was applied at the high rate $(500 \mathrm{~kg} / \mathrm{fed}$.) in the first season 2015 with significant differences with the other treatments. The corresponding mean values for the second season in this respect were 9.3 and $2.01 \mathrm{ton} / \mathrm{fed}$. at the recommended rate of nitrogen fertilizer and 10.0 and $1.8 \mathrm{ton} / \mathrm{fed}$. at the high rate of nitrogen fertilizer. Potassium carbonate gave higher values than those recorded due to using mono-potassium phosphate which scored the lowest plant yields. 
Table 11. Effect of mineral salts applied as foliar spray at two nitrogen rates on fresh weight of herb (ton/fed.) for three cuts of basil plants naturally infected by downy mildew during two growing seasons 2015 and 2016 under field conditions

\begin{tabular}{|c|c|c|c|c|c|c|c|c|c|c|c|}
\hline \multirow{3}{*}{ Treatment } & \multirow{3}{*}{ 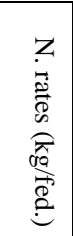 } & \multicolumn{10}{|c|}{ Fresh weight of herb (ton/fed.) } \\
\hline & & \multicolumn{5}{|c|}{ Season 2015} & \multicolumn{5}{|c|}{ Season 2016} \\
\hline & & $\begin{array}{l}1^{\text {st }} \\
\text { cut }\end{array}$ & $\begin{array}{l}2^{\text {nd }} \\
\text { cut }\end{array}$ & $\begin{array}{l}3^{\text {rd }} \\
\text { cut }\end{array}$ & Mean & $\begin{array}{l}\text { Dverall } \\
\text { mean }\end{array}$ & $\begin{array}{l}1^{\text {st }} \\
\text { cut }\end{array}$ & $\begin{array}{l}2^{\text {nd }} \\
\text { cut }\end{array}$ & $\begin{array}{l}3^{\text {rd }} \\
\text { cut }\end{array}$ & Mean & $\begin{array}{l}\text { Overall } \\
\text { mean }\end{array}$ \\
\hline \multirow{2}{*}{$\begin{array}{c}\text { Di-potassium } \\
\text { phosphate } \\
\left(\mathrm{k}_{2} \mathrm{HPO}_{4}\right)\end{array}$} & 400 & 6.8 & 8.7 & 10.3 & 8.6 & \multirow{3}{*}{8.8} & 7.4 & 9.2 & 11.3 & 9.3 & \multirow{3}{*}{9.7} \\
\hline & 500 & 7.2 & 9.0 & 10.7 & 9.0 & & 7.9 & 10.2 & 11.8 & 10.0 & \\
\hline \multicolumn{2}{|l|}{ Mean } & 7.0 & 8.8 & 10.5 & --- & & 7.7 & 9.7 & 11.6 & --- & \\
\hline \multirow{2}{*}{$\begin{array}{c}\text { Mono-potassium } \\
\text { phosphate } \\
\left(\mathrm{KH}_{2} \mathrm{PO}_{4}\right)\end{array}$} & 400 & 5.3 & 6.8 & 8.1 & 6.7 & \multirow{3}{*}{6.9} & 5.8 & 7.8 & 8.5 & 7.4 & \multirow{3}{*}{7.6} \\
\hline & 500 & 5.5 & 7.1 & 9.0 & 7.2 & & 6.2 & 8.1 & 9.2 & 7.8 & \\
\hline \multicolumn{2}{|l|}{ Mean } & 5.4 & 6.9 & 8.5 & --- & & 6.0 & 8.0 & 8.9 & --- & \\
\hline \multirow{2}{*}{$\begin{array}{c}\text { Potassium } \\
\text { carbonate } \\
\left(\mathrm{K}_{2} \mathrm{CO}_{3}\right)\end{array}$} & 400 & 5.7 & 7.7 & 8.9 & 7.4 & \multirow{3}{*}{7.6} & 6.1 & 8.0 & 9.7 & 7.9 & \multirow{3}{*}{8.2} \\
\hline & 500 & 6.0 & 8.0 & 9.5 & 7.8 & & 6.6 & 8.7 & 9.9 & 8.4 & \\
\hline \multicolumn{2}{|l|}{ Mean } & 5.9 & 7.8 & 9.2 & --- & & 6.3 & 8.3 & 9.8 & --- & \\
\hline \multirow{2}{*}{ Stone $50 \%$} & 400 & 8.1 & 10.2 & 12.4 & 10.2 & \multirow{3}{*}{10.5} & 8.9 & 11.0 & 13.4 & 11.1 & \multirow{3}{*}{11.3} \\
\hline & 500 & 8.7 & 10.6 & 12.9 & 10.7 & & 9.5 & 11.6 & 13.6 & 11.6 & \\
\hline \multicolumn{2}{|l|}{ Mean } & 8.4 & 10.4 & 12.7 & --- & & 9.2 & 11.3 & 13.5 & --- & \\
\hline \multirow{2}{*}{ Control } & 400 & 3.2 & 3.7 & 4.4 & 3.8 & \multirow{3}{*}{3.9} & 2.9 & 3.4 & 4.1 & 3.5 & \multirow{3}{*}{3.7} \\
\hline & 500 & 3.4 & 3.9 & 4.7 & 4.0 & & 3.3 & 3.8 & 4.4 & 3.9 & \\
\hline \multicolumn{2}{|l|}{ Mean } & 3.3 & 3.8 & 4.5 & --- & & 3.1 & 3.6 & 4.9 & --- & \\
\hline Mean of & 400 & 5.8 & 7.4 & 8.8 & 7.2 & \multirow{3}{*}{--- } & 6.2 & 7.9 & 9.4 & 7.8 & \multirow{3}{*}{---} \\
\hline nitrogen rates & 500 & 6.2 & 7.7 & 9.3 & 7.7 & & 6.7 & 8.5 & 9.8 & 8.3 & \\
\hline \multicolumn{2}{|c|}{ Overall mean } & 6.0 & 7.6 & 9.1 & --- & & 6.5 & 8.2 & 9.6 & --- & \\
\hline \multicolumn{2}{|c|}{ L.S.D. at $5 \%$ for: } & & $\mathrm{T} \times \mathrm{N}$ & \multicolumn{2}{|c|}{$=\mathrm{ns}$} & & \multicolumn{3}{|c|}{$\mathrm{T} \times \mathrm{N}$} & $=\mathrm{ns}$ & \\
\hline Treatments $(\mathrm{T})$ & $=0.2$ & & $\mathrm{~T} \times \mathrm{C}$ & $=0$ & .36 & & $\mathrm{~T}=0.2$ & & & $=0.34$ & \\
\hline Nitrogen rates & $N)=0$. & & $\mathrm{N} \times \mathrm{C}$ & $=\mathrm{n}$ & & & $\mathrm{N}=0$. & & & $=\mathrm{ns}$ & \\
\hline Cuttings ( C ) & $=0.1$ & & $\mathrm{~T} \times \mathrm{N}$ & $\mathrm{C}=\mathrm{n}$ & & & $\mathrm{C}=0$. & & $\mathrm{N} \times \mathrm{C}$ & $=\mathrm{ns}$ & \\
\hline
\end{tabular}


Table 12. Effect of mineral salts applied as foliar spray at two nitrogen rates on dry weight of herb (ton/fed.) for three cuts of basil plants naturally infected by downy mildew during two growing seasons 2015 and 2016 under field conditions

\begin{tabular}{|c|c|c|c|c|c|c|c|c|c|c|c|}
\hline \multirow{3}{*}{ Treatment } & \multirow{3}{*}{ 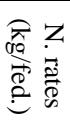 } & \multicolumn{10}{|c|}{ Herb dry weight (ton/fed.) } \\
\hline & & \multicolumn{5}{|c|}{ Season 2015} & \multicolumn{5}{|c|}{ Season 2016} \\
\hline & & \begin{tabular}{|l|}
$1^{\text {st }}$ \\
cut \\
\end{tabular} & $\begin{array}{l}2^{\text {nd }} \\
\text { cut }\end{array}$ & $\begin{array}{l}3^{\text {rd }} \\
\text { cut }\end{array}$ & Mean & $\begin{array}{c}\text { Pverall } \\
\text { mean }\end{array}$ & $\begin{array}{l}1^{\text {st }} \\
\text { cut }\end{array}$ & $\begin{array}{l}2^{\text {nd }} \\
\text { cut }\end{array}$ & $\begin{array}{l}3^{\text {rd }} \\
\text { cut }\end{array}$ & Mean & $\begin{array}{c}\text { Overall } \\
\text { mean }\end{array}$ \\
\hline \multirow{2}{*}{$\begin{array}{l}\text { Di-potassium phosphate } \\
\qquad\left(\mathrm{k}_{2} \mathrm{HPO}_{4}\right)\end{array}$} & 400 & 1.6 & 1.8 & 2.0 & 1.8 & \multirow{3}{*}{1.7} & 1.8 & 2.1 & 2.3 & 2.1 & \multirow{3}{*}{1.9} \\
\hline & 500 & 1.5 & 1.7 & 1.8 & 1.6 & & 1.6 & 1.8 & 2.1 & 1.8 & \\
\hline \multicolumn{2}{|l|}{ Mean } & 1.5 & 1.7 & 1.9 & --- & & 1.7 & 1.9 & 2.2 & --- & \\
\hline \multirow{2}{*}{$\begin{array}{c}\text { Mono-potassium } \\
\text { phosphate }\left(\mathrm{KH}_{2} \mathrm{PO}_{4}\right)\end{array}$} & 400 & 1.2 & 1.4 & 1.7 & 1.4 & \multirow{3}{*}{1.3} & 1.3 & 1.7 & 1.8 & 1.6 & \multirow{3}{*}{1.5} \\
\hline & 500 & 1.1 & 1.3 & 1.40 & 1.3 & & 1.2 & 1.5 & 1.7 & 1.5 & \\
\hline \multicolumn{2}{|l|}{ Mean } & 1.2 & 1.3 & 1.5 & --- & & 1.3 & 1.6 & 1.7 & --- & \\
\hline \multirow{2}{*}{$\begin{array}{l}\text { Potassium carbonate } \\
\qquad\left(\mathrm{K}_{2} \mathrm{CO}_{3}\right)\end{array}$} & 400 & 1.3 & 1.6 & 1.8 & 1.6 & \multirow{3}{*}{1.5} & 1.4 & 1.8 & 2.0 & 1.7 & \multirow{3}{*}{1.7} \\
\hline & 500 & 1.2 & 1.5 & 1.6 & 1.4 & & 1.3 & 1.6 & 1.8 & 1.6 & \\
\hline \multicolumn{2}{|l|}{ Mean } & 1.3 & 1.5 & 1.7 & --- & & 1.3 & 1.7 & 1.9 & --- & \\
\hline \multirow{2}{*}{ Stone $50 \%$} & 400 & 2.0 & 2.3 & 2.5 & 2.2 & \multirow{3}{*}{2.2} & 2.2 & 2.4 & 2.7 & 2.4 & \multirow{3}{*}{2.4} \\
\hline & 500 & 1.8 & 2.1 & 2.3 & 2.0 & & 2.0 & 2.3 & 2.5 & 2.3 & \\
\hline \multicolumn{2}{|l|}{ Mean } & 1.9 & 2.2 & 2.4 & --- & & 2.1 & 2.4 & 2.6 & --- & \\
\hline \multirow{2}{*}{ Control } & 400 & 0.8 & 1.0 & 1.1 & 1.0 & \multirow{3}{*}{0.9} & 0.8 & 0.9 & 1.1 & 0.9 & \multirow{3}{*}{0.8} \\
\hline & 500 & 0.7 & 0.7 & 0.8 & 0.7 & & 0.6 & 0.7 & 0.7 & 0.7 & \\
\hline \multicolumn{2}{|l|}{ Mean } & 0.7 & 0.9 & 0.9 & --- & & 0.7 & 0.8 & 0.9 & --- & \\
\hline \multirow{2}{*}{ Mean of nitrogen rates } & 400 & 1.4 & 1.6 & 1.8 & 1.6 & \multirow{3}{*}{---} & 1.5 & 1.8 & 2.0 & 1.8 & \multirow{3}{*}{---} \\
\hline & 500 & 1.2 & 1.5 & 1.6 & 1.4 & & 1.3 & 1.6 & 1.8 & 1.6 & \\
\hline \multicolumn{2}{|l|}{ Overall mean } & 1.32 & 1.53 & 1.69 & --- & & 1.4 & 1.7 & 1.9 & --- & \\
\hline \multicolumn{2}{|c|}{$\begin{array}{ll}\text { L.S.D. at } 5 \% \text { for: } & \\
\text { Treatments }(\mathrm{T}) & =0.02 \\
\text { Nitrogen rates }(\mathrm{N}) & =0.01 \\
\text { Cuttings }(\mathrm{C}) & =0.02\end{array}$} & \multicolumn{5}{|c|}{$\begin{array}{ll}\mathrm{T} \times \mathrm{N} & =0.03 \\
\mathrm{~T} \times \mathrm{C} & =0.04 \\
\mathrm{~N} \times \mathrm{C} & =0.02 \\
\mathrm{~T} \times \mathrm{N} \times \mathrm{C} & =0.05\end{array}$} & \multicolumn{2}{|c|}{$\begin{array}{l}\mathrm{T}=0.02 \\
\mathrm{~N}=0.02 \\
\mathrm{C}=0.02\end{array}$} & $\begin{array}{l}\mathrm{T} \times \mathrm{N} \\
\mathrm{T} \times \mathrm{C} \\
\mathrm{N} \times \mathrm{C} \\
\mathrm{T} \times \mathrm{N}\end{array}$ & $\begin{aligned} & =0 \\
& =0 . \\
& =0 . \\
C & =0 .\end{aligned}$ & \\
\hline
\end{tabular}

Among all compounds used in this study, chitosan, di-potassium phosphate and potassium silicate in addition to the fungicide Stone $50 \%$ were selected due to their higher effect in controlling basil downy mildew to evaluate their effect on essential oil yields and induction of enzymes activities. Data in Table 13 indicate that all the selected treatments significantly increased essential oil yield/ $100 \mathrm{gm}$ fresh herb than the untreated control at the two rates of nitrogen fertilizer during the two experimental seasons. Among the three tested compounds, chitosan was the most effective treatment yielding the highest increases in the total oil yield by 55.1 \& $38.6 \%$ in the first season and $65.3 \& 57.7$ in the second season at the two rates of nitrogen fertilizer, respectively, followed by di-potassium phosphate salt and the antitranspirant potassium silicate which provided the least oil yields. 
Table 13. Effect of chitosan, di-potassium phosphate, potassium silicate and Stone $50 \%$ as foliar spray at two nitrogen rates on essential oil yield $(\mathrm{ml}) / 100 \mathrm{gm}$ fresh herb during two seasons 2015 and 2016 under field conditions

\begin{tabular}{|c|c|c|c|c|c|c|c|c|c|c|c|}
\hline \multirow{3}{*}{ 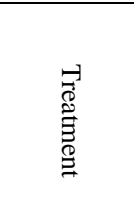 } & \multirow{3}{*}{ 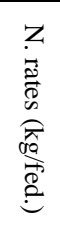 } & \multicolumn{10}{|c|}{ Essential oil yield $(\mathrm{ml}) / 100 \mathrm{gm}$ fresh herb } \\
\hline & & \multicolumn{5}{|c|}{ Season of 2015} & \multicolumn{5}{|c|}{ Season of 2016} \\
\hline & & $\begin{array}{l}1^{\text {st }} \\
\text { cut }\end{array}$ & $\begin{array}{l}2^{\text {nd }} \\
\text { cut }\end{array}$ & $\begin{array}{l}3^{\text {rd }} \\
\text { cut }\end{array}$ & 물 & 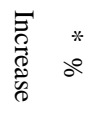 & $\begin{array}{l}1^{\text {st }} \\
\text { cut }\end{array}$ & $\begin{array}{l}2^{\text {nd }} \\
\text { cut }\end{array}$ & $\begin{array}{l}3^{\text {rd }} \\
\text { cut }\end{array}$ & $\stackrel{\overrightarrow{0}}{\stackrel{\partial}{ٍ}}$ & 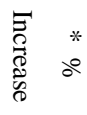 \\
\hline \multirow{2}{*}{ Chitosan } & 400 & 0.2 & 0.2 & 0.3 & 0.7 & 55.1 & 0.2 & 0.3 & 0.3 & 0.7 & 65.3 \\
\hline & 500 & 0.1 & 0.1 & 0.1 & 0.4 & 38.6 & 0.1 & 0.1 & 0.2 & 0.5 & 57.7 \\
\hline \multirow{2}{*}{$\begin{array}{c}\text { Di- } \\
\text { potassium } \\
\text { phosphate }\end{array}$} & 400 & 0.2 & 0.2 & 0.2 & 0.6 & 49.1 & 0.2 & 0.2 & 0.3 & 0.7 & 62.3 \\
\hline & 500 & 0.1 & 0.1 & 0.1 & 0.4 & 27.0 & 0.1 & 0.1 & 0.2 & 0.5 & 52.1 \\
\hline \multirow{2}{*}{$\begin{array}{l}\text { Potassium } \\
\text { silicate }\end{array}$} & 400 & 0.1 & 0.1 & 0.1 & 0.4 & 22.5 & 0.1 & 0.1 & 0.2 & 0.5 & 44.7 \\
\hline & 500 & 0.1 & 0.1 & 0.1 & 0.3 & 12.9 & 0.1 & 0.1 & 0.1 & 0.4 & 40.5 \\
\hline \multirow{2}{*}{$\begin{array}{l}\text { Stone } \\
50 \%\end{array}$} & 400 & 0.2 & 0.2 & 0.3 & 0.7 & 59.2 & 0.3 & 0.3 & 0.3 & 0.8 & 69.0 \\
\hline & 500 & 0.1 & 0.1 & 0.2 & 0.5 & 42.5 & 0.1 & 0.2 & 0.2 & 0.6 & 60.7 \\
\hline \multirow{2}{*}{ Control } & 400 & 0.1 & 0.1 & 0.1 & 0.3 & --- & 0.1 & 0.1 & 0.1 & 0.3 & --- \\
\hline & 500 & 0.1 & 0.1 & 0.1 & 0.3 & --- & 0.0 & 0.1 & 0.1 & 0.2 & --- \\
\hline \multicolumn{2}{|c|}{ L.S.D. at $5 \%$ for: } & & \multicolumn{2}{|c|}{$\mathrm{T} \times \mathrm{N}$} & \multicolumn{2}{|c|}{$=0.012$} & \multicolumn{4}{|c|}{$\mathrm{T} \times \mathrm{N}$} & \\
\hline \multicolumn{2}{|c|}{ Treatments $(\mathrm{T})$} & $=0.003$ & \multicolumn{2}{|c|}{$\mathrm{T} \times \mathrm{C}$} & \multicolumn{2}{|c|}{$=0.012$} & \multicolumn{2}{|c|}{$\mathrm{T}=0.015$} & $\mathrm{~T} \times \mathrm{C}$ & \multicolumn{2}{|c|}{$=0.026$} \\
\hline \multicolumn{3}{|c|}{ Nitrogen rates $(\mathrm{N})=0.009$} & \multicolumn{2}{|c|}{$\mathrm{N} \times \mathrm{C}$} & \multicolumn{2}{|c|}{$=0.012$} & \multicolumn{2}{|c|}{$\mathrm{N}=0.009$} & $\mathrm{~N} \times \mathrm{C}$ & \multicolumn{2}{|c|}{$=0.016$} \\
\hline \multicolumn{2}{|c|}{ Cuttings ( C ) } & 0.002 & \multicolumn{4}{|c|}{$\mathrm{T} \times \mathrm{N} \times \mathrm{C}=0.029$} & \multicolumn{2}{|c|}{$\mathrm{C}=0.006$} & \multicolumn{3}{|c|}{$\mathrm{T} \times \mathrm{N} \times \mathrm{C}=0.036$} \\
\hline
\end{tabular}

*Increase relative to the control.

Results in Table 14 reveal that all treatments increased the enzymes activities at the two nitrogen rates. The highest increase in PAL and POD activity was obtained with fungicide Stone $50 \%$ followed by chitosan treatment, being 55.7 and $94.8 \%$ \& 49.7 and $76.9 \%$ at the recommended rate of nitrogen and reached 54.8 and $85.9 \%$ \& 22.6 and $71.1 \%$ at the high rate of nitrogen, respectively. Di-potassium phosphate treatment showed moderate effect. Meanwhile, potassium silicate was the least effective one. 
Table 14. Enzyme activities in basil plants as affected by chitosan, di-potassium phosphate, potassium silicate and Stone $50 \%$ treatments applied as foliar spray at two nitrogen rates under field conditions

\begin{tabular}{|c|c|c|c|c|c|}
\hline \multirow[t]{2}{*}{ Treatment } & \multirow{2}{*}{$\begin{array}{l}\text { N. rates } \\
\text { (kg/fed.) }\end{array}$} & \multicolumn{2}{|c|}{$\begin{array}{c}\text { Phenylalanine ammonia- } \\
\text { lyase (PAL) }\end{array}$} & \multicolumn{2}{|c|}{$\begin{array}{l}\text { Peroxidase } \\
\quad(\text { POD) }\end{array}$} \\
\hline & & Activity & $* \%$ Increase & Activity & $* \%$ Increase \\
\hline \multirow{2}{*}{ Chitosan } & 400 & 9.925 & 49.7 & 0.052 & 76.9 \\
\hline & 500 & 4.657 & 22.6 & 0.038 & 71.1 \\
\hline \multirow{2}{*}{$\begin{array}{l}\text { Di- } \\
\text { potassium } \\
\text { phosphate }\end{array}$} & 400 & 7.701 & 35.2 & 0.043 & 72.1 \\
\hline & 500 & 3.838 & 6.1 & 0.031 & 64.5 \\
\hline \multirow{2}{*}{$\begin{array}{l}\text { Potassium } \\
\text { silicate }\end{array}$} & 400 & 5.853 & 14.7 & 0.038 & 68.4 \\
\hline & 500 & 3.721 & 3.1 & 0.017 & 35.3 \\
\hline \multirow{2}{*}{ Stone $50 \%$} & 400 & 11.263 & 55.7 & 0.232 & 94.8 \\
\hline & 500 & 7.968 & 54.8 & 0.078 & 85.9 \\
\hline \multirow{2}{*}{ Control } & 400 & 4.992 & --- & 0.012 & --- \\
\hline & 500 & 3.604 & --- & 0.011 & --- \\
\hline
\end{tabular}

* Increase relative to the control.

\section{D i s c u s s i o n}

Plants respond to pathogen attack or elicitor treatments by activating a wide variety of protective mechanisms designed to prevent pathogen replication and spreading. The defense mechanisms include the fast production of reactive oxygen species (De Gara et al., 2003), alterations in the cell wall constitution, accumulation of antimicrobial secondary metabolites known as phytoalexins (Agrios, 2005), activation and/or synthesis of defense peptides and proteins (Castro and Fontes, 2005). In various plant species, resistance can be induced with elicitors such as salicylic acid, bion and chitosan against a wide range of pathogens (Sharathchandra et al., 2004 and Amin et al., 2007). In the present study, it was found that treatment of basil plants with these inducers as foliar spray efficiently reduced susceptibility to downy mildew caused by $P$. belbahrii under field conditions, in a way similar to that of Stone $50 \%$. Applications of chitosan at $0.05 \%$ followed by salicylic acid at 25 $\mathrm{mM}$ were the most effective in this concern. The least effect was found with bion $\left(50 \mathrm{mg} \mathrm{L}^{-1}\right)$ treatment.

There are numerous reports concerning the protective effects of chitosan against downy mildew infection in a range of crops (Manjunatha et al., 2008 and El-Mougy et al., 2014). The reduction of disease severity in plants by chitosan application may be referred to its property as a hydrophobic material, thus creating a low water potential in infected leaves which prevented spore germination, infection and growth when applied before infection as well as, its ability to reduce esterase secretion by pathogens (Hsieh and Huang, 1999). Southerton and Deverall (1990) added that application of elicitors like chitosan resulted in accumulation of osmiophilic masses in the intercellular spaces of reacting host cells. So, the invading fungal cells coated by this osmiophilic material causing frequently pronounced disorganization such as plasmalemma retraction thus preventing pathogen penetration. Another explanation 
may be referred to the role of elicitors in induction of local and/or SAR in plants against invasion of the pathogen. In this concern, SAR was reported in a number of crops due to chitosan application (Bhaskarareddy et al., 1999 and Barka et al., 2004). Several reports indicated also that application of elicitors as chitosan, salicylic acid stimulates the accumulation of signal molecule as jasmonic acid, salicylic acid, hydrogen peroxide, reactive oxygen species and protein kinases, all of which play crucial role in intracellular signalling pathways (Atia et al., 2005). A pioneering study demonstrated that the application of exogenous salicylic acid or chitosan induces the synthesis of pathogenesis related proteins (PRs) and partial resistance to pathogens such as $\beta$-1,3-glucanase, chitinase and PR proteins (Raucher et al., 1999 and Atia et al., 2005).

Exogenous application of salicylic acid has been shown to move systemically through plants, resulting in the expression of a set of defense genes that are activated by pathogen infection (Lu et al., 2006). The development of acquired resistance by salicylic acid may be attributed at least partly, to the SA-induced phenylalanine ammonia lyase (PAL) gene expression and activation (Wen et al., 2005).

On the other hand, application of mineral salts (mono- \& di-potassium phosphate and potassium carbonate) and anti-transpirants (green miracle, potassium silicate, aluminum silicate (kaolin) and silicon dioxide) to the highly susceptible sweet basil plants as foliar spray at weekly intervals before infection by downy mildew significantly controlled the disease and decreased its severity at an unimportant level under field conditions, which consequently improved plant growth and increased fresh and dry weights of basil herbs as well as essential oil yield. Di-potassium phosphate and potassium silicate appeared to be the most effective in this respect. Studies on other plants support our results (Mosa, 1997 and Yildirim et al., 2002). Di-potassium phosphate penetrates the fungal cell and disturbs the balance of potassium and causes the disintegration of conidial walls (Ziv and Zitter, 1992). In addition, it could provide systemic resistance by forming crystallized insoluble compounds with $\mathrm{Ca}^{2+}$ inside the healthy plant cells (Gottstein and Kuc, 1989). Orober et al. (2002) showed that phosphate-mediated resistance induction in cucumber was associated with localized cell death, preceded by a rapid generation of superoxide and hydrogen peroxide. They also detected local and systemic increases in levels of free and conjugated salicylic acid following phosphate application. In barley, phosphate-mediated induction of resistance was associated with increased activities of phenylalanine ammonia-lyase, peroxidase and lipoxygenase (Mitchell and Walters, 2004).

For the protective efficacy of silicates, formation of thick and wide cells as a result of silica accumulation in the epidermal cells plays a role in resistance of the plant against the causal agent (Kuc and Hammerschmidt, 1995). Heintz and Blaich (1990) showed that silicate could accumulate inside the healthy neigh boring cells infected with $U$. necator in such a quantity that they can provide resistance against it. In addition to its protective efficacy, silicates also showed post infection inhibitive efficacy. In this case, silicates inhibit the conidial dispersion and as a result the conidial germination, by covering the colony present on the surface of the plant. Furthermore, silicates can inhibit conidial germination by increasing the $\mathrm{pH}(\mathrm{pH}=10)$ 
(Yildirim and Onogur, 2001). Oh (1997) reported that the required $\mathrm{pH}$ for germination of conidia of $U$. necator was 5 in laboratory conditions.

Various mechanisms for the protected plants with coating polymers have been suggested (Han, 1990 and Zekaria-Oren et al., 1991). The effect of film forming anti-transpirants may be similar to those of the natural cuticle layer in defence against pathogen. In this respect, Zekaria-Oren et al. (1991) mentioned that polymers of film forming anti-transpirants provide either an impenetrable surface associated with their thickness or are resistance to enzymatic degrading.

In this study, application of nitrogen fertilizer above the recommended rates significantly increased disease severity of basil downy mildew. This result is in line with Hoffland et al. (2000); Zarafi et al. (2005) and dos Santos et al. (2009). The N supply influences branching and leaf expansion, which together determine the size of the canopy produced. Large canopies with high shoot densities may be more conducive to spore transfer and pathogen infection than sparse canopies (Walters and Bingham, 2007). Olesen et al. (2003a) found that the severity of both powdery mildew and septoria leaf spot was enhanced by increasing rates of $\mathrm{N}$ fertilizer that associated with an increased leaf $\mathrm{N}$ concentration at flag leaf emergence (Olesen $e t$ al., 2003b). These workers also obtained positive, but weaker, correlations between disease severity and crop canopy size, suggesting that leaf $\mathrm{N}$ concentration was more important for disease development than canopy size (Olesen et al., 2003b). According to Hoffland et al. (2000), susceptibility to disease is the outcome of the interaction between two factors: (a) the value of the plant as a source of nutrients and energy to the pathogen and (b) the presence of secondary host metabolites (defense compounds) that prevent pathogen growth, development or multiplication. These factors are affected differentially by $\mathrm{N}$ supply.

In conclusion, the incorporation of chitosan, di-potassium phosphate and potassium silicate in an integrated management programs should bring positive results, both in terms of reducing disease intensity and consequently, the amount of fungicides used.

\section{References}

Abd-Alla, F.M.F. 2004. Studies on powdery mildew disease affecting Solanaceous plants. M.Sc. Thesis, Fac. Agric., Cairo Univ., Cairo.

Agrios, G.N. 2005. Plant Pathology. $5^{\text {th }}$ Ed. Academic Press, San Diego, USA.

Amin, A.A.; Rashad EL-Sh., M. and EL-Abagy, H.M.H. 2007. Physiological effect of indole-3-butyric acid and salicylic acid on growth, yield and chemical constituents of onion plants. J. Appl. Sci. Res., 3(11): 1554-1563.

Atia, M.M.M.; Buchenauer, H.; Aly, A.Z. and Abou-Zaid, M.I. 2005. Antifungal activity of chitosan against Phytophthora infestans and cultivation of defense mechanisms in tomato to late blight. Biol. Agric. Horti., 23(2): 175-197. 
Barka, E.A.; Eullaffroy, P.; Clement, C. and Vernet, G. 2004. Chitosan improves development, and protects Vitis vinifera L. against Botrytis cinerea. Plant Cell Rep., 22: 608-614.

Baysal, Ö.; Soylu, E.M. and Soylu, S. 2003. Induction of defence-related enzymes and resistance by the plant activator acibenzolar-S-methyl in tomato seedlings against bacterial canker caused by Clavibacter michiganensiss sp. Michiganensis. Plant Pathol., 52: 747-753.

Belbahri, L.; Calmin, G.; Pawlowski, J. and Lefort, F. 2005. Phylogenetic analysis and real time PCR detection of a presumably undescribed Peronospora species on sweet basil and sage. Mycol. Res., 109:1276-1287.

Ben-Naim, Y.; Falach, L. and Cohen, Y. 2015. Resistance against basil downy mildew in Ocimum species. Phytopathology, 105: 778-785.

Bhaskarareddy, M.V.; Arul, J.; Angers, P. and Couture, L. 1999. Chitosan treatment of wheat seeds induces resistance to Fusarium graminearum and improves seed quality. J. Agric. Food Chem., 47: 1208-1216.

Bowen, P.; Menzies, J. and Ehret, D. 1992. Soluble silicon sprays inhibit powdery mildew development on grape leaves. J. Am. Soc. Hort. Sci., 117: 906-912.

Castro, M.S. and Fontes, W. 2005. Plant defense and antimicrobial peptides. Protein Peptide Letters, 12: 11-16.

Cohen, Y.; Vaknin, M.; Ben-Naim, Y.; Rubin, A.E.; Galperin, M.; Silverman, D.; Bitton, S. and Adler, U. 2013. First report of the occurrence and resistance to mefenoxam of Peronospora belbahrii, causal agent of downy mildew of basil (Ocimum basilicum) in Israel. Plant Dis., 97(5): 692.

Dallagnol, L.J.; Rodrigues, F.A.; Tanaka, F.A.O.; Amorim, L. and Camargo, L.E.A. 2012. Effect of potassium silicate on epidemic components of powdery mildew on melon. Plant Pathol., 61: 323-330.

De Gara, L.; de Pinto, M.C. and Tommasi, F. 2003. The antioxidant systems vis-àvis reactive oxygen species during plant pathogen interaction. Plant Physiol. Biochem., 41: 863-870.

dos Santos, G.R.; de Castro Neto, M.D.; de Almeida, H.S.M.; Ramos, L.N.; Sarmento, R.A.; de O Lima, S. and Erasmo, E.A.L. 2009. Effect of nitrogen doses on disease severity and watermelon yield. Horticultura Brasileira, 27: 330-334.

El-Mougy, Nehal S.; Abdel-Kader, M.M. and Lashin, S.M. 2014. Protective foliar approaches against downy and powdery mildews of cantaloupe under plastic houses conditions. Int. J. Engin. Innovative Technol., 3(9): 324-329.

Epstein, E. 2009. Silicon: its manifold roles in plants. Annal. Appl. Biol., 155:155160. 
Fawe, A.; Menzies, J.G.; Cherif, M. and Bélanger, R.R. 2001. Silicon and disease resistance in dicotyledons. In: Datnoff L.E, Snyder G.H., Korndorfer G.H., (Eds). "Silicon in Agriculture". Amsterdam, the Netherlands: Elsevier, 159-169.

Garibaldi, A.; Minuto, G.; Bertelli, D. and Gullino, M.L., 2004. Seed transmission of Peronospora sp. of basil. J. Plant Dis. Plant Prot., 111(5): 465-469.

Gilardi, G.; Demarchi, S.; Garibaldi, A. and Gullino, M.L. 2013. Management of downy mildew of sweet basil (Ocimum basilicum) caused by Peronospora belbahrii by means of resistance inducers, fungicides, biocontrol agents and natural products. Phytoparasitica 41: 59-72.

Gilardi, G.; Pintore, I.; Demarchi, S.; Gullino, M.L. and Garibaldi, A. 2015.Seed dressing to control downy mildew of basil. Phytoparasitica, 43: 531-539.

Gottstein, H. and Kuc, J. 1989: Induction of systemic resistance to anthracnose in cucumber by phosphates. Phytopathology, 79: 176-179.

Gregory, N.F. 2009. Downy mildew on basil. University of Deleware. Cooperative Extension, PP-47 (c. a. Homa, 2013).

Guenther, E. 1961. The Essential Oils. D. Van Norstand Inc. NY, USA. 569p.

Gullino, M.L.; Gilardi, G. and Garibaldi, A. 2009. Chemical control of downy mildew on lettuce and basil under greenhouse. Commun. Agric. Applied Biol. Sci., 74(3): 933-940.

Haggag, Wafaa M. 2002. Control of downy mildew on protected cucumber plants with film forming anti-transpirants. J. Biol. Sci., 2(6): 403-407.

Han, J.S. 1990. Use of anti-transpirant epidermal coatings for plant protection in China. Plant Dis., 74: 263-266.

Heintz, C. and Blaich, R. 1990. Ultrastructural and histochemical studies on interactions between Vitis vinifera L. and Uncinula necator (Schw.) Burr. New Phytol., 115: 107-117.

Hilal, A.A. and Ghebrial, Eman W. 2014. Occurrence of downy mildew (Peronospora belbahrii) of sweet basil (Ocimum basilicum L.) in Egypt. Egypt. J. Phytopathol., 42(2): 197-198.

Hoffland, E.; Jeger, M.J. and van Beusichem, M.L. 2000. Effect of nitrogen supply rate on disease resistance in tomato depends on the pathogen. Plant Soil, 218: 239-247.

Homa, K. 2013. Evaluating fungicide efficacy, plant varietal resistance and leaf morphology in developing control strategies for basil downy mildew (Peronospora belbahrii). M.Sc.Thesis, Rutgers, New Jersey, 1-216.

Homa, K.; Barney, W.P.; Ward, D.L.; Wyenandt, C.A. and Simon, J.E. 2014. Evaluation of fungicides for the control of Peronospora belbahrii on sweet basil in New Jersey. Plant Dis., 98: 1561-1566. 
Hsieh, T.F. and Huang, J.W. 1999. Effect of film-forming polymers on control of lily leaf blight caused by Botrytis elliptica. Eur. J. Pl. Pathol., 105: 501-508.

Hussain, A.I.; Anwar, F.; Hussain Sherazi, S.T. and Przybylski, R. 2008. Chemical composition, antioxidant and antimicrobial activities of basil (Ocimum basilicum) essential oils depends on seasonal variations. Food Chem., 108: 986-995.

Kanto, T.; Miyoshi, A.; Ogawa, T.; Maekawa, K. and Aino, M. 2006. Suppressive effect of liquid potassium silicate on powdery mildew of strawberry in soil. $J$. Gen. Plant Pathol., 72: 137-142.

Koroch, A.R.; Wang, W.; Michael, T.P.; Dudai, N.; Simon, J.E. and Belanger, F.C. 2010. Estimation of nuclear DNA content of cultivated Ocimum species by using flow cytometry. Isr. J. Plant Sci., 58: 183-189.

Kuc, J. and Hammerschmidt, R. 1995: Induced resistance to disease in plants: Developments in Plant Pathology. vol. 4, Kluwer Academic Publishers, The Netherlands, 4, $192 \mathrm{pp}$.

Lea, P.J. and Azevedo, R.A. 2006. Nitrogen use efficiency. 1. Uptake of nitrogen from the soil. Annals Applied Biol., 149: 243-247.

Lea, P.J.; Sodek, L.; Parry, M.A.J.; Shewry, P.R. and Halford, N.G. 2007. Asparagine in plants. Annals Applied Biol., 150: 1-26.

Leadbeater, A. and Gisi, U. 2010. The challenges of chemical control of plant diseases. In U. Gisi, I. Chet, \& M.L. Gullino (Eds.), Recent Developments In Management of Plant Diseases. Dordrecht: Springer.pp. 3-17.

Liang, Y.C.; Chen, Q.; Liu, Q.; Zhang, W.H. and Ding, R.X. 2003. Exogenous silicon ( $\mathrm{Si}$ ) increases antioxidant enzyme activity and reduces lipid peroxidation in roots of salt-stressed barley (Hordeum vulgare L.). J. Pl. Physiol., 160: 11571164.

Lopez, A.M.Q. and Lucas, J.A. 2002. Effects of plant defence activators on anthracnose disease of cashew. Eur. J. Pl. Pathol., 108: 409-420.

Lu, Z.X.; Gaudet, D.; Puchalski, B.; Despins, T.; Frick, M. and Laroche, A. 2006. Inducers of resistance reduce common bunt infection in wheat seedlings while differentially regulating defense-gene expression. Physiol. Mol. Pl. Pathol., 67: 138-148.

Manjunatha, G.; Roopa, K.S.; Geetha, N. Prashanth and Shekar Shetty, H. 2008. Chitosan enhances disease resistance in pearl millet against downy mildew caused by Sclerosporagraminicolaand defence-related enzyme activation. Pest Manag. Sci., 64: 1250-1257.

McGrath, M.T. 2009. Expect and prepare for downy mildew in basil. Rutgers Plant and Pest Advisory, Vegetable Crops Edition, 15(11): 1-2.

Mersha, Z.; Zhang, S.; Fu, Y.; Mo, X.; Raid, R.N. and Hau, B. 2013. Efficacy of acibenzolar-S-methyl and $\beta$-aminobutyric acid for control of downy mildew in 
greenhouse grown basil and peroxidase activity in response to treatment with these compounds. J. Phytopathol., 161: 154-164.

Mersha, Z.; Zhang S. and Raid, R.N. 2012. Evaluation of systemic acquired resistance inducers for control of downy mildew on basil. Crop Prot., 40: 83-90.

Mitchell, A.F. and Walters, D.R. 2004. Potassium phosphate induces systemic protection in barley to powdery mildew infection. Pest Manag. Sci., 60: 126-134.

Mosa, A.A. 1997. Effect of foliar application of phosphates on cucumber powdery mildew. Ann. Agric. Sci., 42: 241-255.

Nasraoui, B. 1993. Role des films anti-transpirantsdans la phytoprotectioncontre les maladies fongiques. Annals de l'INRAT, 66(1-2): 125-135.

Nasraoui, B.; Barbier, A. and Lepoivre, P. 1996. Effect of three antitranspirant films on Botrytis cinerea activities in vitro. Arab J. Pl. Prot., 14(2): 98-101.

Oh, J.H. 1997. Effects of temperature, relative humidity, $\mathrm{pH}$ and fungicides on sporulation and conidial germination of Uncinula necator. Korean J. Pl. Pathol., 13: $210-214$.

Olesen, J.E.; Jorgensen, L.N.; Petersen, J. and Mortensen, J.V. 2003a. Effects of rate and timing of nitrogen fertilizer on disease control by fungicides in winter wheat. 1. Grain yield and foliar disease control. J. Agric. Sci., Cambridge, 140: 1-13.

Olesen, J.E.; Jorgensen, L.N.; Petersen, J. and Mortensen, J.V. 2003b. Effects of rates and timing of nitrogen fertilizer on disease control by fungicides in winter wheat. 2. Crop growth and disease development. J. Agric. Sci., Cambridge, 140: $15-29$.

Orober, M.; Siegrist, J. and Buchenauer, H. 2002. Mechanisms of phosphateinduced disease resistance in cucumber. Eur. J. Pl. Pathol., 108: 345-353.

Patel, J.S.; Zhang, S. and Costa de Novaes, M.I. 2014. Effect of plant age and acibenzolar-S-methyle on development of downy mildew of basil. Hort Science, 49(11): 1392-1396.

Prapagdee, B.; Kotchadat, K.; Kumsopa, A. and Visarathanonth, N. 2007. The role of chitosan in protection of soybean from sudden death syndrome caused by Fusarium solani f.sp. glycines. Bioresource Technol., 98(7): 1353-1358.

Rauscher, M.; Ádám, A.L.; Wirtz, S.; Guggenheim, R.; Kurt, M. and Deising, H.B. 1999. PR-1 protein inhibits the differentiation of rust infection hyphae in leaves of acquired resistant broad bean. The Plant J., 19(6): 625-633.

Rodrigues, F.A.; McNally, D.J.; Datnoff, L.E.; Jones, J.B.; Labbe, C.; Benhamou, N.; Menzies, J.G. and Bélanger, R.R. 2004. Silicon enhances the accumulation of diterpenoid phytoalexins in rice: a potential mechanism for blast resistance. Phytopathology, 94: 177-183.

Samuels, A.L.; Glass, A.D.M.; Ehret, D.L. and Menzies, J.G. 1991. Distribution of silicon in cucumber leaves during infection by powdery mildew fungus (Sphaerotheca fuliginea). Can. J. Bot., 69(1): 140-146. 
Sharathchandra, R.G.; Niranjan Raj, S.; Shetty, N.P.; Amruthesh, K.N. and Shekar Shetty, H. 2004. A chitosan formulation Elexa induces downy mildew disease resistance and growth promotion in pearl millet. Crop Prot., 23: 881-888.

Simon, J.E.; Quinn, J. and Murray, R.G. 1990. Basil: a source of essential oils,. In: Janick, J. and J.E. Simon (Eds.). "Advances in New Crops". Timber Press, Portland, OR, pp. 484-489

Snedecor, G.W. and Cochran, W.G. 1989. Statistical Methods. $8^{\text {th }}$ ed. Iowa State Univ. Press, Ames, Iowa, USA, 251p.

Southerton, S.G. and Deverall, B.J. 1990. Changes in phenolic acid level in wheat leaves expressing resistance to Puccinia recondite f.sp. tritici. Physiol. Mol. Pl. Pathol., 37:437-450.

Suppakul, P.; Miltz, J.; Sonneveld, K. and Bigger, S.W. 2003.Antimicrobial properties of basil and its possible application in food packaging. J. Agric. Food Chem., 51: 3197-3207.

Thines, M.; Telle, S.; Ploch, S. and Runge, F. 2009.Identity of the downy mildew pathogens of basil, coleus and sage with implications for quarantine measures. Mycol. Res., 113:532-540.

Walters, D.R. and Bingham, I.J. 2007. Influence of nutrition on disease development caused by fungal pathogens: implications for plant disease control. Ann. Appl. Biol., 151: 307-324.

Walters, D.R. and Murray, D.C. 1992. Induction of systemic resistance to rust in Vicia faba by phosphate and EDTA: effects of calcium. Plant Pathol., 41: 444448.

Wen, P.F.; Chen, J.Y.; Kong, W.F.; Pan, Q.H.; Wan, S.B. and Huang, W.D. 2005. Salicylic acid induced the expression of phenylalanine ammonia-lyase gene in grape berry. Plant Sci., 169: 928-934.

Worthington, X.X. 1971. "Enzyme Manual", Worthington Biochemical Corp., Freehold, New Jersey.

Yildirim, I. and Onogur, E. 2001. Effects of alternative substances on conidial germination and appressorium formation of Uncinula necator (Schw.) Burr.In vitro. J. Turkish Phytopathol., 30: 36-46.

Yildirim, I.; Onogur, E. and Irshad, M. 2002. Investigations on the efficacy of some natural chemicals against powdery mildew [Uncinula necator (Schw.) Burr.] of grape. J. Phytopathol., 150: 697-702.

Zarafi, A.B.; Emechebe, A.M.; Akpa, A.D. and Alabi, O. 2005. Effect of fertilizer levels on grain yield, incidence and severity of downy mildew in pearl millet. Arch. Phytopathol. Pl. Prot., 38(1): 11-17.

Zekaria-Oren, J.; Eyal, Z. and Ziv, O. 1991. Effect of film-forming compounds on the development of leaf rust on wheat seedling. Plant Dis., 75: 231-234. 
Ziv, O. and Zitter, T.A. 1992. Effect of bicarbonates and film-forming polymers on cucurbit foliar diseases. Plant Dis., 76: 513-517.

Zucker, M. 1965. Induction of phenylalanine deaminase by light and its reaction to chlorogenic acid synthesis in potato tuber tissue. Plant Physiol., 40: 779-784.

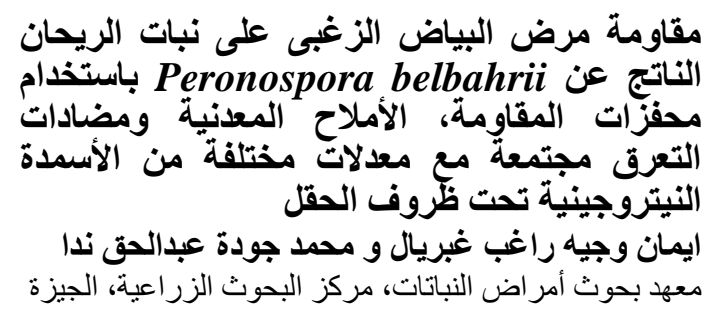

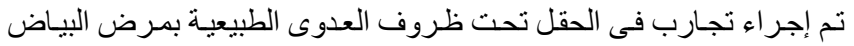

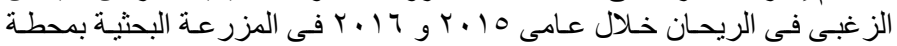

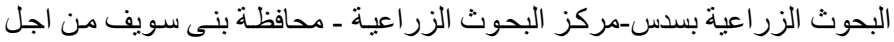

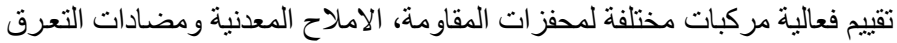

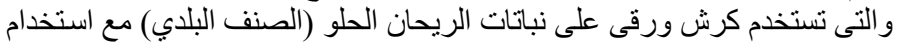

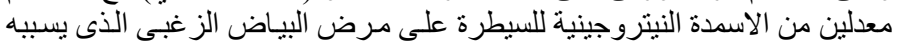

Peronospora belbahrii

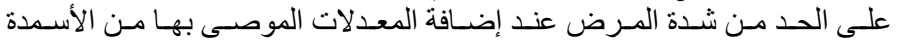

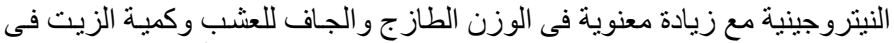

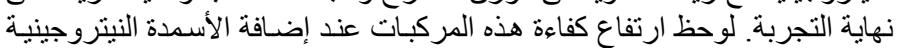

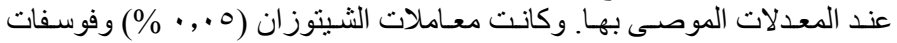

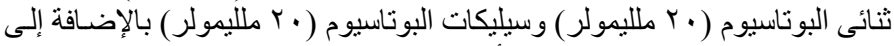

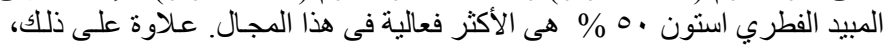

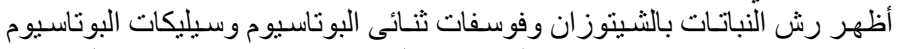

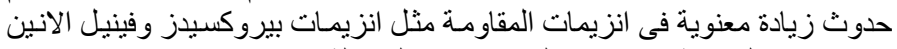

امونيا لايز بالمقارنة مع نباتات الريحان غير الرئات المعاملة. 